\title{
FAST ESTIMATION FROM ABOVE OF THE MAXIMUM WAVE SPEED IN THE RIEMANN PROBLEM FOR THE EULER EQUATIONS*
}

\author{
JEAN-LUC GUERMOND ${ }^{\dagger}$ AND BOJAN POPOV ${ }^{\dagger}$
}

\begin{abstract}
This paper is concerned with the construction of a fast algorithm for computing the maximum speed of propagation in the Riemann solution for the Euler system of gas dynamics with the co-volume equation of state. The novelty in the algorithm is that it stops when a guaranteed upper bound for the maximum speed is reached with a prescribed accuracy. The convergence rate of the algorithm is cubic and the bound is guaranteed for gasses with the co-volume equation of state and the heat capacity ratio $\gamma$ in the range $(1,5 / 3]$.
\end{abstract}

Key words. Euler system of gas dynamics, co-volume equation of state, maximum speed of propagation, Riemann problem

AMS subject classifications. 65M60, 65M10, 65M15, 35L65

1. Introduction. The objective of this paper is to propose a fast algorithm to estimate from above the maximum wave speed in the Riemann problem for the Euler equations of gas dynamics. This quantity, or an approximation thereof, is used in many numerical methods to approximate the solution of the compressible Euler equations using various representations: finite volumes, discontinuous Galerkin, continuous finite elements, etc., see e.g., Rusanov [19, Eq. (9)] or Harten et al. [13, Eq. (2.6b)]. The motivation for the present work comes from a multidimensional finite element technique recently proposed in Guermond and Popov [11]. This method is explicit and uses continuous finite elements on unstructured grids in any space dimension. The artificial viscosity in the method is defined so that having an upper bound on the maximum speed of propagation in the one-dimensional Riemann problems guaranties that all the entropy inequalities are satisfied and the algorithm is invariant domain preserving in the sense of Chueh et al. [7], Hoff [14], Frid [9], i.e., the density and the internal energy are nonnegative and the specific entropy satisfies a local minimum principle. It is also shown therein that the closer the upper bound on the maximum wave speed the larger the admissible CFL. We stress here that it is not the entire solution of the Riemann problem that is required to ensure the above properties, but only a guaranteed upper bound on the maximum wave speed. Standard Riemann solvers, either approximate or exact, are designed to give an approximation of the solution at the interface, and this in general requires solving for intermediate states in the Riemann fan. This task is far more computationally intensive than estimating the maximum wave speed of the Riemann fan. Note in passing that traditional estimates of the maximum wave speed in ideal gases, which consists of taking $\max \left(\left|u_{L}\right|+a_{L},\left|u_{R}\right|+a_{R}\right)$, where $a$ is the speed of sound and $u$ is the velocity, could either be wrong or be an overestimate thereof (a counterexample is produced in the appendix B), see e.g., Kurganov and Tadmor [16, Eq. (3.2)] or Toro [20, §10.5.1]. In conclusion, we claim that $\max \left(\left|u_{L}\right|+a_{L},\left|u_{R}\right|+a_{R}\right)$ is not an upper bound on the

* This material is based upon work supported in part by the National Science Foundation grant DMS-1217262, by the Air Force Office of Scientific Research, USAF, under grant/contract number FA99550-12-0358, and the Army Research Office, under grant number W911NF-15-1-0517. Draft version, April 8, 2016

${ }^{\dagger}$ Department of Mathematics, Texas A\&M University 3368 TAMU, College Station, TX 77843, USA.

(C) 2016. This manuscript version is made available under the Elsevier user license http://www.elsevier.com/open-access/userlicense/1.0/ 
maximum wave speed, and solving for the intermediate states, as done in traditional Riemann solvers, is expensive and is not necessary to ensure that invariant domains are preserved, as established in $[11,12]$.

The novelty of the present work is the construction of a fast algorithm for computing the maximum wave speed in the Riemann problem for the Euler equations with the co-volume equation of state (which includes ideal gases). One important feature of the algorithm is that it terminates when an upper bound for the maximum speed is obtained with a prescribed tolerance. The algorithm has a cubic convergence rate and the upper bound is guaranteed for gasses with co-volume equation of state and a heat capacity ratio $1<\gamma \leq 5 / 3$. We have obtained $10^{-15}$ accuracy in at most three steps in all the numerical experiments we have done with the proposed algorithm. We stress here that traditional Riemann solvers based on Newton's method on the intermediate pressure $p^{*}$ do not guarantee the upper bound on the maximum wave speed; actually most of these solvers converge to $p^{*}$ from below and thereby underestimate the maximum wave speed. Another original result of the present work is an inexpensive guaranteed upper bound of the maximum wave speed given in Remark 4.3. This estimate is actually used to initialize the iterative algorithm.

This paper is organized as follows. We introduce some notation and collect general statements about the one-dimensional Riemann problem in $\S 2$. The main result of this section is the well known Proposition 2.1. We introduce additional notation in $\S 3$ and recall the expression for the extreme wave speeds of the 1 -wave and the 3 -wave. We introduce the algorithm to compute a guaranteed upper bound on the maximum wave speed in $\S 4$. It is shown in Theorem 4.6 that the algorithm terminates in finite time and delivers a guaranteed upper bound up to any prescribed threshold. An important result that makes the convergence of the method cubic and guarantees the upper bound is stated in Theorem 4.1. The gap condition proved in Lemma 4.8 is essential to prove that the algorithm terminates in finite time. Both Theorem 4.1 and Lemma 4.8 are original to the best of our knowledge. The performance of the algorithm is tested in $\S 5$; it is shown in $\S 5.3$ that when used in a numerical solver for the compressible Euler equations proposed in [11], the overhead induced by the proposed iterative method is minimal. Additional theoretical statements for the covolume equation of state and counter-examples showing that $\max \left(\left|u_{L}\right|+a_{L},\left|u_{R}\right|+a_{R}\right)$ may be sometimes significantly smaller and sometimes significantly bigger than the actual maximum wave speed of the Riemann problem are reported in Appendices A and B. A source code is provided in Appendix C.

2. Preliminaries. We introduce notations and discuss the notion of Riemann problem in this section. The main result is the well known Proposition 2.1. The reader who is already familiar with Riemann problems and Proposition 2.1 can skip this section and go directly to $\S 3$.

2.1. Formulation of the problem. Consider the compressible Euler equations

$$
\partial_{t} \boldsymbol{c}+\nabla \cdot(\boldsymbol{f}(\boldsymbol{c}))=0, \quad \boldsymbol{c}=\left(\begin{array}{c}
\rho \\
\boldsymbol{m} \\
E
\end{array}\right), \quad \boldsymbol{f}(\boldsymbol{c})=\left(\begin{array}{c}
\boldsymbol{m} \\
\boldsymbol{m} \otimes \frac{\boldsymbol{m}}{\rho}+p \mathbb{I} \\
\frac{\boldsymbol{m}}{\rho}(E+p)
\end{array}\right),
$$

where the independent variables are the density $\rho$, the momentum vector field $\boldsymbol{m}$ and the total energy $E$. The velocity vector field $\boldsymbol{u}$ is defined by $\boldsymbol{u}:=\boldsymbol{m} / \rho$ and the internal energy density $e$ by $e:=\rho^{-1} E-\frac{1}{2}\|\boldsymbol{u}\|_{\ell^{2}}^{2}$, where $\|\cdot\|_{\ell^{2}}$ is the Euclidean norm. The quantity $p$ is the pressure. The symbol $\mathbb{I}$ denotes the identity matrix in $\mathbb{R}^{d \times d}$. 
In this paper, we only consider the so-called co-volume gasses obeying the co-volume Equation Of State (EOS),

$$
p(1-b \rho)=(\gamma-1) e \rho,
$$

with $b \geq 0$; the case $b=0$ corresponds to an ideal gas. The constant $b$ is called the co-volume and $\gamma>1$ is the ratio of specific heats. Sometimes, the co-volume EOS is called the Noble-Abel EOS. We refer to Toro [20, Chapter 1.2], Baibuz et al. [1] and Johnston [15] for more details on these EOS and the related thermodynamics.

In the context of the method proposed in Guermond and Popov [11], we consider the following one-dimensional Riemann problem:

$$
\partial_{t} \boldsymbol{c}+\partial_{x}(\boldsymbol{f}(\boldsymbol{c}) \cdot \boldsymbol{n})=0, \quad(x, t) \in \mathbb{R} \times \mathbb{R}_{+}, \quad \boldsymbol{c}(x, 0)=\left\{\begin{array}{ll}
\boldsymbol{c}_{L}, & \text { if } x<0 \\
\boldsymbol{c}_{R}, & \text { if } x>0,
\end{array} .\right.
$$

where $\boldsymbol{n}$ is any unit vector in $S^{d}(0,1)$. The solution to this problem is also invoked in many Riemann-solver-based Godunov type methods, see e.g., Toro and Titarev [21], Bouchut and Morales de Luna [3], Castro et al. [5], Balsara et al. [2]. We stress that we are only interested in estimating from above the maximum wave speed in (2.3). It is shown in [11] that having an upper bound on the maximum speed of propagation of the one-dimensional Riemann problem guaranties that the first-order algorithm described in [11] is invariant domain preserving in the sense of Chueh et al. [7], Hoff [14], Frid [9], and that it satisfies all the entropy inequalities.

The problem (2.3) is hyperbolic, since $\partial_{\rho} p(\rho, s)$ is positive, and the Jacobian of $\boldsymbol{f}(\boldsymbol{c}) \cdot \boldsymbol{n}$ is diagonalizable with real eigenvalues. It is well known in the case of ideal and co-volume gases with $\gamma>1$ that (2.3) has a unique (physical) solution, which we henceforth denote $\boldsymbol{c}\left(\boldsymbol{n}, \boldsymbol{u}_{L}, \boldsymbol{u}_{R}\right)$, see Toro [20, Chapter 4.7].

Note that the admissibility condition for the left and right states is $0<1-$ $b \rho_{L}, 1-b \rho_{R}<1$. A simple but lengthy verification shows that the exact solution of the Riemann problem in all possible cases stays admissible across the entire Riemann fan, that is $1-b \rho>0$. Being unaware of a reference for this result, we give a proof in the appendix for completeness, see Proposition A.1.

2.2. Structure of the Riemann problem. The multidimensional Riemann problem (2.3) was first described in the context of dimension splitting schemes in two space dimensions in Chorin [6, p. 526]. The general case is treated in Colella [8, p. 188], see also Toro [20, Chapter 4.8]. We make a change of basis and introduce $\boldsymbol{t}_{1}, \ldots, \boldsymbol{t}_{d-1}$ so that $\left\{\boldsymbol{n}, \boldsymbol{t}_{1}, \ldots, \boldsymbol{t}_{d-1}\right\}$ forms an orthonormal basis of $\mathbb{R}^{d}$. With this new basis we have $\boldsymbol{m}=\left(m, \boldsymbol{m}^{\perp}\right)^{\top}$, where $m:=\rho u, u:=\boldsymbol{u} \cdot \boldsymbol{n}, \boldsymbol{m}^{\perp}:=\rho\left(\boldsymbol{u} \cdot \boldsymbol{t}_{1}, \ldots, \boldsymbol{u} \cdot \boldsymbol{t}_{d-1}\right):=\rho \boldsymbol{u}^{\perp}$. The projected equations are

$$
\partial_{t} \boldsymbol{c}+\partial_{x}(\boldsymbol{n} \cdot \boldsymbol{f}(\boldsymbol{c}))=\mathbf{0}, \quad \boldsymbol{c}=\left(\begin{array}{c}
\rho \\
m \\
\boldsymbol{m}^{\perp} \\
E
\end{array}\right), \quad \boldsymbol{n} \cdot \boldsymbol{f}(\boldsymbol{c})=\left(\begin{array}{c}
m \\
\frac{1}{\rho} m^{2}+p \\
u \boldsymbol{m}^{\perp} \\
u(E+p)
\end{array}\right) .
$$

Using $\rho, u, \boldsymbol{u}^{\perp}$ and the specific entropy $s$ as dependent variables, the above problem can be rewritten

$$
\left\{\begin{array}{l}
\partial_{t} \rho+\partial_{x}(\rho u)=0 \\
\partial_{t} u+u \partial_{x}(u)+\rho^{-1} \partial_{x} p(\rho, s)=0 \\
\partial_{t} \boldsymbol{u}^{\perp}+u \partial_{x}\left(\boldsymbol{u}^{\perp}\right)=0 \\
\partial_{t} s+u \partial_{x}(s)=0,
\end{array}\right.
$$


and the Jacobian is

$$
\left(\begin{array}{cccc}
u & \rho & \mathbf{0}^{\top} & 0 \\
\rho^{-1} \partial_{\rho} p & u & \mathbf{0}^{\top} & \rho^{-1} \partial_{s} p \\
\mathbf{0} & \mathbf{0} & u \mathbb{I} & \mathbf{0} \\
0 & 0 & \mathbf{0}^{\top} & u
\end{array}\right)
$$

The eigenvalues are $\lambda_{1}=u-\sqrt{\partial_{\rho} p(\rho, s)}$, with multiplicity $1, \lambda_{2}=\cdots=\lambda_{d+1}=$ $u$, with multiplicity $d$, and $\lambda_{d+2}=u+\sqrt{\partial_{\rho} p(\rho, s)}$, with multiplicity 1 . One key observation is that the Jacobian does not depend on $\boldsymbol{m}^{\perp}$, see Toro [20, p. 150]. As a consequence the solution of the Riemann problem with data $\left(\boldsymbol{c}_{L}, \boldsymbol{c}_{R}\right)$, is obtained in two steps.

2.2.1. First step. We solve the one-dimensional Riemann problem

$$
\partial_{t}\left(\begin{array}{c}
\rho \\
m \\
\mathcal{E}
\end{array}\right)+\partial_{x}\left(\begin{array}{c}
m \\
\frac{1}{\rho} m^{2}+p \\
\frac{m}{\rho}(\mathcal{E}+p)
\end{array}\right)=0, \quad \text { with } \quad p(1-b \rho)=(\gamma-1)\left(\mathcal{E}-\frac{m^{2}}{2 \rho}\right)
$$

with data $\boldsymbol{c}_{L}:=\left(\rho_{L}, \boldsymbol{m}_{L} \cdot \boldsymbol{n}, \mathcal{E}_{L}\right)^{\top}, \boldsymbol{c}_{R}:=\left(\rho_{R}, \boldsymbol{m}_{R} \cdot \boldsymbol{n}, \mathcal{E}_{R}\right)^{\top}$, where $\mathcal{E}=E-\frac{1}{2} \frac{\left\|\boldsymbol{m}^{\perp}\right\|_{\ell^{2}}^{2}}{\rho}$. The one-dimensional Riemann problem (2.6) is strictly hyperbolic and all the characteristic fields are either genuinely nonlinear or linearly degenerate. Any Riemann problem of this type with $n$ fields has a unique self-similar weak solution in Lax's form for any initial data such that $\left\|\boldsymbol{u}_{L}-\boldsymbol{u}_{R}\right\|_{\ell^{2}} \leq \delta$, see Lax [18] and Bressan [4, Thm 5.3]. In particular there are $2 n$ numbers

$$
\lambda_{1}^{-} \leq \lambda_{1}^{+} \leq \lambda_{2}^{-} \leq \lambda_{2}^{+} \leq \ldots \leq \lambda_{n}^{-} \leq \lambda_{n}^{+},
$$

defining up to $2 m+1$ sectors (some could be empty) in the $(x, t)$ plane:

$$
\frac{x}{t} \in\left(-\infty, \lambda_{1}^{-}\right), \quad \frac{x}{t} \in\left(\lambda_{1}^{-}, \lambda_{1}^{+}\right), \ldots, \quad \frac{x}{t} \in\left(\lambda_{n}^{-}, \lambda_{n}^{+}\right), \quad \frac{x}{t} \in\left(\lambda_{n}^{+}, \infty\right) .
$$

The Riemann solution is $\boldsymbol{u}_{L}$ in the sector $\frac{x}{t} \in\left(-\infty, \lambda_{1}^{-}\right)$and $\boldsymbol{u}_{R}$ in the last sector $\frac{x}{t} \in\left(\lambda_{n}^{+}, \infty\right)$. The solution in the other sectors is either a constant state or an expansion, see Bressan [4, Chap. 5]. The sector $\lambda_{1}^{-} t<x<\lambda_{n}^{+} t, 0<t$, is henceforth referred to as the Riemann fan. The key result that we are going to use is that there is a maximum speed of propagation $\lambda_{\max }\left(\boldsymbol{n}, \boldsymbol{u}_{L}, \boldsymbol{u}_{R}\right):=\max \left(\left|\lambda_{1}^{-}\right|,\left|\lambda_{n}^{+}\right|\right)$such that for $t \geq 0$ we have

$$
\boldsymbol{u}(x, t)= \begin{cases}\boldsymbol{u}_{L}, & \text { if } x \leq-t \lambda_{\max }\left(\boldsymbol{n}, \boldsymbol{u}_{L}, \boldsymbol{u}_{R}\right) \\ \boldsymbol{u}_{R}, & \text { if } x \geq t \lambda_{\max }\left(\boldsymbol{n}, \boldsymbol{u}_{L}, \boldsymbol{u}_{R}\right)\end{cases}
$$

In the special case of the one dimensional Euler equations of gas dynamics (2.6) with the co-volume EOS, we have $n=3$, the smallness assumption on the Riemann data is not needed, see Toro [20, Chap. 4], and the Riemann fan is composed of three waves only: (i) two genuinely nonlinear waves, $\lambda_{i}^{ \pm}, i \in\{1,3\}$, which are either shocks (in which case $\lambda_{i}^{-}=\lambda_{i}^{+}:=\lambda_{i}$ ) or rarefaction waves; (ii) one linearly degenerate middle wave which is a contact discontinuity, $\lambda_{2}^{-}=\lambda_{2}^{+}=: u^{*}$. 
2.2.2. Second step. We complete the full solution of the Riemann problem (2.4) by determining $\boldsymbol{m}^{\perp}$. We compute $\boldsymbol{m}^{\perp}$ by solving $\partial_{t} \boldsymbol{m}^{\perp}+\partial_{x}\left(u \boldsymbol{m}^{\perp}\right)=0$. The solution is composed of up to four states: $\boldsymbol{m}_{L}^{\perp}, \boldsymbol{m}_{L}^{\perp, *}, \boldsymbol{m}_{R}^{\perp, *}, \boldsymbol{m}_{R}^{\perp}$,

$$
\boldsymbol{m}= \begin{cases}\boldsymbol{m}_{L}^{\perp} & \text { if } x \leq \lambda_{1}^{+} t, \\ \boldsymbol{m}_{L}^{\perp, *} & \text { if } \lambda_{1}^{+} t \leq x \leq \lambda_{2} t, \\ \boldsymbol{m}_{R}^{\perp, *} & \text { if } \lambda_{2} t \leq x \leq \lambda_{3}^{-} t \\ \boldsymbol{m}_{R}^{\perp} & \text { if } \lambda_{3}^{-} t \leq x,\end{cases}
$$

where $\boldsymbol{m}_{L}^{\perp, *}$ is such that $\boldsymbol{m}_{L}^{\perp, *}=\boldsymbol{m}_{L}^{\perp}$ if $\lambda_{1}^{-} \neq \lambda_{1}^{+}$(i.e., if the leftmost wave is a rarefaction) or $\boldsymbol{m}_{L}^{\perp, *}$ is given by the Rankine-Hugoniot condition $u_{L} \boldsymbol{m}_{L}^{\perp}-u^{*} \boldsymbol{m}_{L}^{\perp, *}=$ $\lambda_{1}\left(\boldsymbol{m}_{L}^{\perp}-\boldsymbol{m}_{L}^{\perp, *}\right)$ otherwise (i.e., if the leftmost wave is a shock). Similarly $\boldsymbol{m}_{R}^{\perp, *}$ is computed as follows: $\boldsymbol{m}_{R}^{\perp, *}=\boldsymbol{m}_{R}^{\perp}$ if $\lambda_{3}^{-} \neq \lambda_{3}^{+}$(i.e., the rightmost wave is a rarefaction) or $\boldsymbol{m}_{R}^{\perp, *}$ is given by the Rankine-Hugoniot condition $u_{R} \boldsymbol{m}_{R}^{\perp}-u^{*} \boldsymbol{m}_{R}^{\perp, *}=\lambda_{3}\left(\boldsymbol{m}_{R}^{\perp}-\boldsymbol{m}_{R}^{\perp, *}\right)$ otherwise (i.e., the rightmost wave is a shock). The Rankine-Hugoniot condition is automatically satisfied across the contact wave $u^{*}\left(\boldsymbol{m}_{L}^{\perp, *}-\boldsymbol{m}_{R}^{\perp, *}\right)=\lambda_{2}\left(\boldsymbol{m}_{L}^{\perp, *}-\boldsymbol{m}_{R}^{\perp, *}\right)$ since $u^{*}:=\lambda_{2}$. Note that the solution given in Toro $[20, \S 3.2 .4, \S 4.8]$ is correct only if the two extreme waves (i.e., the 1 -wave and the 3 -wave) are both rarefactions.

2.2.3. Maximum wave speed. The bottom line of the above argumentation is that the organization of the Riemann fan is entirely controlled by the solution of (2.6) and therefore we have

Proposition 2.1. In the case of gases obeying the co-volume equation of state, the maximum wave speed in (2.4) is

$$
\lambda_{\max }\left(\boldsymbol{c}_{L}, \boldsymbol{c}_{R}\right)=\max \left(\left(\lambda_{1}^{-}\left(\boldsymbol{c}_{L}, \boldsymbol{c}_{R}\right)\right)_{-},\left(\lambda_{3}^{+}\left(\boldsymbol{c}_{L}, \boldsymbol{c}_{R}\right)\right)_{+}\right),
$$

$z_{-}=\max (0,-z), z_{+}=\max (0, z)$, and $\lambda_{1}^{-}\left(\boldsymbol{c}_{L}, \boldsymbol{c}_{R}\right), \lambda_{3}^{+}\left(\boldsymbol{c}_{L}, \boldsymbol{c}_{R}\right)$ are the two extreme wave speeds in the Riemann problem (2.6) with data $\left(\boldsymbol{c}_{L}, \boldsymbol{c}_{R}\right)$.

The goal of this paper is to propose a fast algorithm to estimate accurately from above the maximum speed of propagation $\lambda_{\max }\left(\boldsymbol{c}_{L}, \boldsymbol{c}_{R}\right)$. This program is achieved by estimating $\lambda_{1}^{-}\left(\boldsymbol{c}_{L}, \boldsymbol{c}_{R}\right)$ from below and $\lambda_{3}^{+}\left(\boldsymbol{c}_{L}, \boldsymbol{c}_{R}\right)$ from above.

3. Computation of $\lambda_{1}^{-}\left(\boldsymbol{c}_{L}, \boldsymbol{c}_{R}\right)$ and $\lambda_{3}^{+}\left(\boldsymbol{c}_{L}, \boldsymbol{c}_{R}\right)$. We compute $\lambda_{1}^{-}\left(\boldsymbol{c}_{L}, \boldsymbol{c}_{R}\right)$ and $\lambda_{3}^{+}\left(\boldsymbol{c}_{L}, \boldsymbol{c}_{R}\right)$ in this section. Most of the material is quoted from Toro [20, Chap. 4]. We henceforth abuse the notation by using the symbol $\boldsymbol{c}$ for the primitive variables, i.e., $\boldsymbol{c}=(\rho, u, p)^{\top}$, instead of using the conservative variables as defined above.

3.1. Riemann data with vacuum. When vacuum is present in the left or in the right state, the Riemann solution is composed of two waves only: one rarefaction on the non-vacuum side and a contact discontinuity on the vacuum side. The left and right maximum wave speeds are given by the following expressions:

$$
\lambda_{1}^{-}\left(\boldsymbol{c}_{L}, \boldsymbol{c}_{R}\right)=u_{L}-a_{L}, \quad \lambda_{2}\left(\boldsymbol{c}_{L}, \boldsymbol{c}_{R}\right)=u_{L}+\frac{2 a_{L}\left(1-b \rho_{L}\right)}{\gamma-1},
$$

with $\boldsymbol{c}_{L}=\left(\rho_{L}, u_{L}, p_{L}\right)^{\top}$ and $\boldsymbol{c}_{R}=\left(0, u_{R}, 0\right)^{\top}$ for vacuum on the right, and

$$
\lambda_{2}\left(\boldsymbol{c}_{L}, \boldsymbol{c}_{R}\right)=u_{R}-\frac{2 a_{R}\left(1-b \rho_{R}\right)}{\gamma-1}, \quad \lambda_{3}^{+}\left(\boldsymbol{c}_{L}, \boldsymbol{c}_{R}\right)=u_{R}+a_{R},
$$

with $\boldsymbol{c}_{L}=\left(0, u_{L}, 0\right)^{\top}$ and $\boldsymbol{c}_{R}=\left(\rho_{R}, u_{R}, p_{R}\right)^{\top}$ for vacuum on the left. See $\S 4.6 .1, \S 4.6 .2$ and $\S 4.7 .1$ in Toro [20] for the details. Then $\lambda_{\max }\left(\boldsymbol{c}_{L}, \boldsymbol{c}_{R}\right)=\max \left(\left(\lambda_{1}^{-}\right)_{-},\left(\lambda_{2}\right)_{+}\right)$in the first case and $\lambda_{\max }\left(\boldsymbol{c}_{L}, \boldsymbol{c}_{R}\right)=\max \left(\left(\lambda_{2}\right)_{-},\left(\lambda_{3}^{+}\right)_{+}\right)$in the second case. 
3.2. Riemann data without vacuum. We now restrict ourselves to the case where both states, $\boldsymbol{c}_{L}$ and $\boldsymbol{c}_{R}$, are not vacuum states, i.e., $\rho_{L}, \rho_{R}>0, e_{L}, e_{R} \geq 0$, and admissible states $1-b \rho_{L}, 1-b \rho_{R}>0$. A simple but lengthy verification shows that the exact solution of the Riemann problem in all possible cases is admissible for all times, that is $1-b \rho>0$. This result is proved in Proposition A.1 in the appendix A. In the numerical applications we have in mind, see Guermond and Popov [11], only averages of the exact Riemann solution over the entire Riemann fan are invoked. Therefore, if the left and the right states are admissible non-vacuum states, the average of the exact Riemann solution over the Riemann fan is an admissible non-vacuum state even if the exact solution contains vacuum. Since the method in [11] preserves all the convex invariant sets of the Riemann problem and the set $\{\rho>0,1-b \rho>0\}$ is convex and invariant, we conclude that if the initial data is admissible and does not contain a vacuum state, the numerical method in [11] produces only admissible non-vacuum states at every time step.

The no vacuum condition $0<\rho_{L}, \rho_{R}$ and the admissibility conditions $0<1-$ $b \rho_{L}, 1-b \rho_{R}, 0 \leq e_{L}, e_{R}$ imply that $p_{L}, p_{R} \in[0, \infty)$. Then the local sound speed is given by $a_{Z}=\sqrt{\frac{\gamma p_{Z}}{\rho_{Z}\left(1-b \rho_{Z}\right)}}$ where the index $Z$ is either $L$ or $R$. We introduce the following notation $A_{Z}:=\frac{2\left(1-b \rho_{Z}\right)}{(\gamma+1) \rho_{Z}}, B_{Z}:=\frac{\gamma-1}{\gamma+1} p_{Z}$ and the functions

$$
\begin{aligned}
\phi(p) & :=f(p, L)+f(p, R)+u_{R}-u_{L} \\
f(p, Z) & := \begin{cases}\left(p-p_{Z}\right)\left(\frac{A_{Z}}{p+B_{Z}}\right)^{\frac{1}{2}} & \text { if } p \geq p_{Z}, \\
\frac{2 a_{Z}\left(1-b \rho_{Z}\right)}{\gamma-1}\left(\left(\frac{p}{p_{Z}}\right)^{\frac{\gamma-1}{2 \gamma}}-1\right) & \text { if } p<p_{Z},\end{cases}
\end{aligned}
$$

where again $Z$ is either $L$ or $R$. Let $a_{Z}^{0}$ be the speed of sound for the ideal gas, and let $A_{Z}^{0}, B_{Z}^{0}, \phi^{0}(p)$ and $f^{0}(p, Z)$ be the above defined quantities in the ideal gas case, i.e., we take $b=0$ in all definitions. Then we have that $a_{Z}=\frac{a_{Z}^{0}}{\sqrt{1-b \rho_{Z}}}$, $f(p, Z)=f^{0}(p, Z) \sqrt{1-b \rho_{Z}}$ and

$$
\phi(p)=f^{0}(p, L) \sqrt{1-b \rho_{L}}+f^{0}(p, R) \sqrt{1-b \rho_{R}}+u_{R}-u_{L} .
$$

It is shown in Toro [20, Chapter 4.3.1] (see also Bressan [4, Eq. (5.36)]) that the functions $f^{0}(p, L), f^{0}(p, R) \in C^{2}\left(\mathbb{R}_{+} ; \mathbb{R}\right)$ are monotone increasing and concave down. Therefore the function $\phi(p) \in C^{2}\left(\mathbb{R}_{+} ; \mathbb{R}\right)$ is also monotone increasing and concave down. It can also be shown that the weak third derivative is non-negative and locally bounded. Observe that $\phi(0)=u_{R}-u_{L}-\frac{2 a_{L}^{0} \sqrt{1-b \rho_{L}}}{\gamma-1}-\frac{2 a_{R}^{0} \sqrt{1-b \rho_{R}}}{\gamma-1}$. Therefore, $\phi$ has a unique positive root if and only if $\phi(0)<0$, i.e.,

$$
u_{R}-u_{L}<\frac{2 a_{L}^{0} \sqrt{1-b \rho_{L}}}{\gamma-1}+\frac{2 a_{R}^{0} \sqrt{1-b \rho_{R}}}{\gamma-1}
$$

This is the well known non-vacuum condition in the case of ideal gas ( $b=0$ above), see Toro $\left[20,(4.40)\right.$, p. 127]. We henceforth denote this root by $p^{*}$, i.e., $\phi\left(p^{*}\right)=0$. We conventionally set $p^{*}=0$ if (3.6) does not hold. It can be shown that, whether there is formation of vacuum or not, the two extreme wave speeds $\lambda_{1}^{-}\left(\boldsymbol{c}_{L}, \boldsymbol{c}_{R}\right)$ and 
$\lambda_{3}^{+}\left(\boldsymbol{c}_{L}, \boldsymbol{c}_{R}\right)$ enclosing the Riemann fan are

$$
\begin{aligned}
& \lambda_{1}^{-}\left(\boldsymbol{c}_{L}, \boldsymbol{c}_{R}\right)=u_{L}-a_{L}\left(1+\frac{\gamma+1}{2 \gamma}\left(\frac{p^{*}-p_{L}}{p_{L}}\right)_{+}\right)^{\frac{1}{2}}, \\
& \lambda_{3}^{+}\left(\boldsymbol{c}_{L}, \boldsymbol{c}_{R}\right)=u_{R}+a_{R}\left(1+\frac{\gamma+1}{2 \gamma}\left(\frac{p^{*}-p_{R}}{p_{R}}\right)_{+}\right)^{\frac{1}{2}},
\end{aligned}
$$

where $z_{+}:=\max (0, z)$.

Remark 3.1. (Two rarefaction waves) Note that if $\phi\left(p_{L}\right)>0$ then $p_{L}>p^{*}$, by monotonicity of $\phi$, thereby implying that $\lambda_{1}^{-}\left(u_{L}, u_{R}\right)=u_{L}-a_{L}$ in this case. Similarly if $\phi\left(p_{R}\right)>0$, then $p_{R}>p^{*}$ and $\lambda_{3}^{+}\left(u_{L}, u_{R}\right)=u_{R}+a_{R}$. This observation means that there is no need to compute $p^{*}$ to estimate $\lambda_{\max }\left(\boldsymbol{c}_{L}, \boldsymbol{c}_{R}\right)$ when $\phi\left(\min \left(p_{L}, p_{R}\right)\right)>0$. This happens when the two extreme waves are rarefactions. Noticing that $p^{*}$ does not need to be evaluated in this case is important since traditional techniques to compute $p^{*}$ in this situation may require a large number of (unnecessary) iterations, see Toro [20, p. 128]. This is particularly true when (3.6) is violated, since in this case there is a formation of a vacuum state.

4. Accurate estimation of $\lambda_{\max }$ from above. In this section we present an algorithm for computing an accurate lower bound on $\lambda_{1}^{-}\left(\boldsymbol{c}_{L}, \boldsymbol{c}_{R}\right)$ and an accurate upper bound on $\lambda_{3}^{-}\left(\boldsymbol{c}_{L}, \boldsymbol{c}_{R}\right)$. This is done by estimating accurate lower and upper bounds on the intermediate pressure state $p^{*}$.

4.1. Elementary waves. If the exact solution of the Riemann problem contains two rarefaction waves, i.e., $p^{*} \leq \min \left(p_{L}, p_{R}\right)$, no computation of $p^{*}$ is needed, see Remark 3.1.

Let us define $p_{\min }:=\min \left(p_{L}, p_{R}\right), p_{\max }:=\max \left(p_{L}, p_{R}\right)$ and let us assume that $\phi\left(p_{\min }\right) \leq 0$. Note that if $\phi\left(p_{\min }\right)=0$, then $p^{*}=p_{\min }$ and nothing needs to be done. We now assume that $p^{*}>p_{\min }$ and we define

$$
\begin{array}{r}
\phi_{R}(p)=\frac{2 a_{L}\left(1-b \rho_{L}\right)}{\gamma-1}\left(\left(\frac{p}{p_{L}}\right)^{\frac{\gamma-1}{2 \gamma}}-1\right)+\frac{2 a_{R}\left(1-b \rho_{R}\right)}{\gamma-1}\left(\left(\frac{p}{p_{R}}\right)^{\frac{\gamma-1}{2 \gamma}}-1\right) \\
+u_{R}-u_{L}
\end{array}
$$

Note that $\phi_{R}$ is monotone increasing and concave down. We also have the following result (see also left panel in Figure 4.1).

TheOrem 4.1. Assume $\gamma \in\left(1, \frac{5}{3}\right]$. For any $p \geq 0$, the graph of $(p, \phi(p))$ is above the graph of $\left(p, \phi_{R}(p)\right)$; more precisely, $\phi_{R}(p)=\phi(p)$ for all $p \in\left[0, p_{\min }\right]$ and $\phi_{R}(p)<\phi(p)$ for all $p \in\left(p_{\min }, \infty\right)$.

Proof. Note that the two curves $(p, \phi(p))$ and $\left(p, \phi_{R}(p)\right)$ coincide if $p \leq p_{\min }$ because both are the sum of the two rarefaction curves and the constant $u_{R}-u_{L}$. If $p_{\min }<p \leq p_{\max }$ the $(p, \phi(p))$ curve is the sum of one rarefaction curve, one shock curve starting from $p=p_{\min }$ and the constant $u_{R}-u_{L}$. If $p \geq p_{\max }$ the $(p, \phi(p))$ curve is the sum of two shock curves and the constant $u_{R}-u_{L}$, see (3.4). Now we invoke Lemma 4.2 twice to complete the proof, once with $p^{0}=p_{\min }\left(\rho^{0}\right.$ being the associated density) and once with $p^{0}=p_{\max }\left(\rho^{0}\right.$ being the associated density).

Lemma 4.2. Let $p^{0}>0$ and $\rho^{0}$ such that $0<1-b \rho^{0}<1$. Assume that $1<\gamma \leq \frac{5}{3}$. 
We define the shock curve passing through $p^{0}$ by

$$
f_{S}(p)=\left(p-p^{0}\right) \sqrt{\frac{2}{(\gamma+1) \rho^{0}}}\left(p+\frac{\gamma-1}{\gamma+1} p^{0}\right)^{-\frac{1}{2}} \sqrt{1-b \rho^{0}}
$$

and the rarefaction curve by

$$
f_{R}(p)=\frac{2 \sqrt{\frac{\gamma p^{0}}{\rho^{0}}}}{\gamma-1}\left(\left(\frac{p}{p^{0}}\right)^{\frac{\gamma-1}{2 \gamma}}-1\right) \sqrt{1-b \rho^{0}}
$$

Then $f_{R}(p)<f_{S}(p)$ for any $p>p^{0}$ and $f_{R}\left(p^{0}\right)=f_{S}\left(p^{0}\right)$, i.e., the shock curve is above the rarefaction curve.

Proof. We rescale both the shock and the rarefaction curves for $p \geq p^{0}$ by introducing the variable $x=p / p^{0}$ and set $f_{S}(p)=\tilde{f}_{S}(x), f_{R}(p)=\tilde{f}_{R}(x)$. The two curves now are now

$$
\tilde{f}_{S}(x)=\frac{\sqrt{2 p^{0}\left(1-b \rho^{0}\right)}}{\sqrt{(\gamma+1) \rho^{0}}} \frac{(x-1)}{\sqrt{x+\frac{\gamma-1}{\gamma+1}}}, \quad \tilde{f}_{R}(x)=\frac{2 \sqrt{\gamma p^{0}\left(1-b \rho^{0}\right)}}{(\gamma-1) \sqrt{\rho^{0}}}\left(x^{\frac{\gamma-1}{2 \gamma}}-1\right) .
$$

Note that $\tilde{f}_{S}(1)=\tilde{f}_{R}(1)=0$. We assume from now on that $x>1$ and we want to show that $\tilde{f}_{S}(x)>\tilde{f}_{R}(x)$ for any $x>1$ if $\gamma \leq 5 / 3$. Instead of proving this directly, we consider the function

$$
g(x)=\frac{\rho^{0}(\gamma+1)\left(x+\frac{\gamma-1}{\gamma+1}\right)}{2 p^{0}\left(1-b \rho^{0}\right)\left(x^{\frac{\gamma-1}{2 \gamma}}-1\right)^{2}}\left(\tilde{f}_{S}(x)^{2}-\tilde{f}_{R}(x)^{2}\right)
$$

and we will show that $g(x)>0$ for any $x>1$ if $\gamma \leq 5 / 3$. We change variable again and set $y:=x^{\frac{\gamma-1}{2 \gamma}}$ with $g(x)=\tilde{g}(y)$. Then setting $y^{\alpha}=x$ with $\alpha=2+\frac{2}{\gamma-1}$ we have

$$
\tilde{g}(y)=\left(\frac{y^{\alpha}-1}{y-1}\right)^{2}-\alpha\left((\alpha-1) y^{\alpha}+1\right) .
$$

We rearrange the terms in (4.2) to get

$$
\tilde{g}(y)=\left(\frac{y^{\alpha}-1}{y-1}-\frac{1}{2} \alpha(\alpha-1)(y-1)\right)^{2}-\frac{1}{4} \alpha^{2}(\alpha-1)^{2}(y-1)^{2}-\alpha^{2}, \quad \forall y>1 .
$$

Using a Taylor expansion of $y^{\alpha}$ at $y=1$ for any $y>1$ and $\alpha \geq 4$, we obtain the inequality

$$
\frac{y^{\alpha}-1}{y-1} \geq \alpha+\frac{1}{2} \alpha(\alpha-1)(y-1)+\frac{1}{6} \alpha(\alpha-1)(\alpha-2)(y-1)^{2} .
$$

Using this inequality in (4.2), we have that

$$
\tilde{g}(y) \geq\left(\alpha+\frac{1}{6} \alpha(\alpha-1)(\alpha-2)(y-1)^{2}\right)^{2}-\frac{1}{4} \alpha^{2}(\alpha-1)^{2}(y-1)^{2}-\alpha^{2}
$$

which is equivalent to

$$
\tilde{g}(y) \geq\left(\frac{1}{6} \alpha(\alpha-1)(\alpha-2)(y-1)^{2}\right)^{2}+\frac{\alpha^{2}(\alpha-1)}{12}(\alpha-5)(y-1)^{2} .
$$


Therefore, we infer that $\tilde{g}(y)>0$ for any $y>1$ provided $\alpha \geq 5$. Note that the condition $\alpha \in[5, \infty)$ is equivalent to $\gamma \in\left(1, \frac{5}{3}\right]$. Hence we conclude that $\tilde{f}_{S}(x)>\tilde{f}_{R}(x)$ for any $x>1$ if $1<\gamma \leq 5 / 3$.

Remark 4.1. (Physical range of $\gamma$ ) Note that the $\gamma$-law usually assumes that $\gamma=$ $\frac{M+2}{M}$, where $M \geq 3$ is the number of degrees of freedom of the molecules composing the gas. We have $M=3$ for monatomic gases and $M=5$ for diatomic gases. Therefore, the physical range of $\gamma$ for $M \in[3, \infty)$ is $\gamma \in\left(1, \frac{5}{3}\right]$, which happens to be exactly the range of application of Lemma 4.2.

Remark 4.2. (Non physical range of $\gamma$ ) In the non-physical range $\gamma>5 / 3$ it can be shown via Taylor series argument that there is $x_{0}=x_{0}(\gamma)>1$ such that $f_{S}\left(x_{0}\right)<$ $f_{R}\left(x_{0}\right)$. Therefore, the statements of both Theorem 4.1 and Lemma 4.2 are false if $\gamma \in(5 / 3,+\infty)$.

4.2. The algorithm to estimate $\lambda_{\max }$. We now continue with the construction of an algorithm for computing the intermediate pressure $p^{*}$, keeping in mind that the quantity we are after is $\lambda_{\max }$. Recall that we only consider the case $\phi\left(p_{\min }\right)<$ 0 . Both functions $\phi$ and $\phi_{R}$ are strictly monotone increasing and $\lim _{p \rightarrow \infty} \phi(p)=$ $\lim _{p \rightarrow \infty} \phi_{R}(p)=+\infty$, therefore they each have a unique zero. The zero of $\phi$ is $p^{*}$ and we denote the zero of $\phi_{R}$ by $\tilde{p}^{*}$. The zero of $\phi_{R}$ is easy to compute

$$
\tilde{p}^{*}=\left(\frac{a_{L}^{0} \sqrt{1-b \rho_{L}}+a_{R}^{0} \sqrt{1-b \rho_{R}}-\frac{\gamma-1}{2}\left(u_{R}-u_{L}\right)}{a_{L}^{0} \sqrt{1-b \rho_{L}} p_{L}^{-\frac{\gamma-1}{2 \gamma}}+a_{R}^{0} \sqrt{1-b \rho_{R}} p_{R}^{-\frac{\gamma-1}{2 \gamma}}}\right)^{\frac{2 \gamma}{\gamma-1}}
$$

and is referred to in the literature as the two-rarefaction approximation to $p^{*}$, see for example equation (4.103) in Toro [20, Chapter 4.7.2].

Lemma 4.3. We have $p^{*}<\tilde{p}^{*}$ in the physical range of $\gamma, 1<\gamma \leq \frac{5}{3}$.

Proof. This is an easy consequence of Theorem 4.1. To the best of our knowledge, this result, which is important to establish accurate a priori error estimates on $p^{*}$, is new. $\mathrm{c}$

We now propose an iterative process composed of two algorithms that constructs two sequences $\left(p_{1}^{k}, p_{2}^{k}\right)_{k \geq 0}$ such that $p_{1}^{k} \leq p^{*} \leq p_{2}^{k}$ for all $k \geq 0$ and $\lim _{k \rightarrow+\infty} p_{1}^{k}=$ $p^{*}=\lim _{k \rightarrow+\infty} p_{2}^{k}$. Before going through the details of the algorithms, we describe a stopping criterion that guarantees accuracy on the maximum wave speed. Let $k \geq 0$ and assume that $p_{1}^{k} \leq p^{*} \leq p_{2}^{k}$. Then using (3.7)-(3.8), we have $v_{11}^{k} \leq \lambda_{1}^{-} \leq v_{12}^{k}$ and $v_{31}^{k} \leq \lambda_{3}^{+} \leq v_{32}^{k}$, where

$$
\begin{array}{ll}
v_{11}^{k}=u_{L}-a_{L}\left(1+\frac{\gamma+1}{2 \gamma}\left(\frac{p_{2}^{k}-p_{L}}{p_{L}}\right)_{+}\right)^{\frac{1}{2}}, & v_{12}^{k}=u_{L}-a_{L}\left(1+\frac{\gamma+1}{2 \gamma}\left(\frac{p_{1}^{k}-p_{L}}{p_{L}}\right)_{+}\right)^{\frac{1}{2}} \\
v_{31}^{k}=u_{R}+a_{R}\left(1+\frac{\gamma+1}{2 \gamma}\left(\frac{p_{1}^{k}-p_{R}}{p_{R}}\right)_{+}\right)^{\frac{1}{2}}, & v_{32}^{k}=u_{R}+a_{R}\left(1+\frac{\gamma+1}{2 \gamma}\left(\frac{p_{2}^{k}-p_{R}}{p_{R}}\right)_{+}\right)^{\frac{1}{2}}
\end{array}
$$

and we have $\lambda_{\min }^{k}<\lambda_{\max } \leq \lambda_{\max }^{k}$ for any $k \geq 0$ with the definitions

$$
\lambda_{\max }^{k}:=\max \left(\left(v_{32}^{k}\right)_{+},\left(v_{11}^{k}\right)_{-}\right), \quad \lambda_{\min }^{k}:=\left(\max \left(\left(v_{31}^{k}\right)_{+},\left(v_{12}^{k}\right)_{-}\right)\right)_{+} .
$$

Lemma 4.4. Let $\epsilon>0$, and assume that $p_{1}^{k} \leq p^{*} \leq p_{2}^{k}$ and $\lambda_{\min }^{k}>0$ then

$$
\left(\frac{\lambda_{\max }^{k}}{\lambda_{\min }^{k}}-1 \leq \epsilon\right) \Longrightarrow\left(\left|\lambda_{\max }^{k}-\lambda_{\max }\right| \leq \epsilon \lambda_{\max }\right)
$$


Proof. The inequalities $p_{1}^{k} \leq p^{*} \leq p_{2}^{k}$ together with (4.4) and (4.5) implies that $\lambda_{\min }^{k}<\lambda_{\max } \leq \lambda_{\max }^{k}$, which in turn gives

$$
\begin{aligned}
\frac{\left|\lambda_{\max }^{k}-\lambda_{\max }\right|}{\lambda_{\max }} & =\frac{\lambda_{\max }^{k}-\lambda_{\max }}{\lambda_{\max }}=\frac{\lambda_{\max }^{k}}{\lambda_{\max }}-1 & \text { since } \lambda_{\max }<\lambda_{\max }^{k} \\
& \leq \frac{\lambda_{\max }^{k}}{\lambda_{\min }^{k}}-1 \leq \epsilon & \text { since } \lambda_{\min }^{k}<\lambda_{\max } .
\end{aligned}
$$

This completes the proof.

The process that we propose consists of two algorithms: the first one generates an initial guess $\left(p_{1}^{0}, p_{2}^{0}\right)$ such that $p_{1}^{0}<p^{*}<p_{2}^{0}$; the second one iterates until the stopping criterion described in Lemma 4.4 is satisfied. The initialization is described in Algorithm 1.

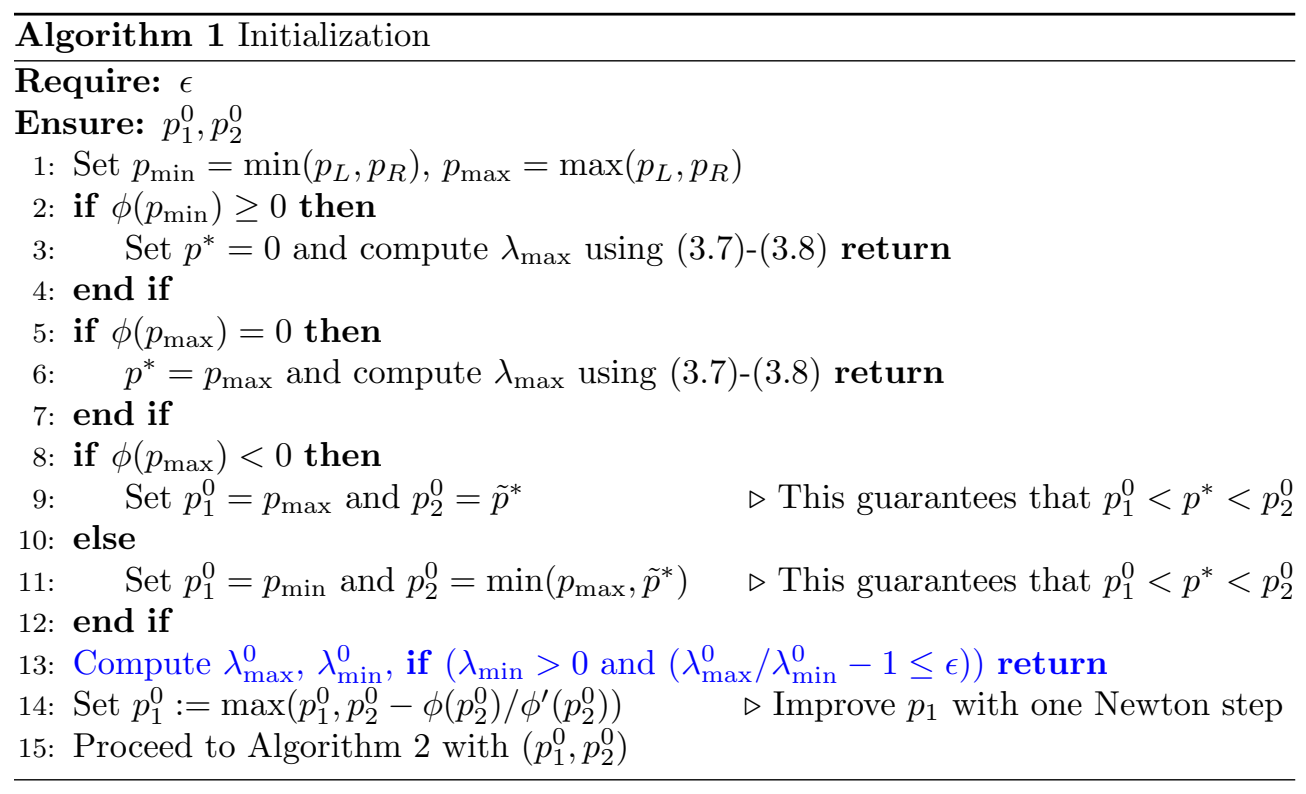

Very often the initialization process described in Algorithm 1 is accurate enough to be used in applications. Line 13 in the algorithm checks the accuracy of the initialization in terms of relative error on $\lambda_{\max }$.

Remark 4.3. (Non-iterative estimate) It is possible to use Algorithm 1 alone without iterating since $p_{2}^{0}$ is an upper bound on $p^{*}$ and

$$
v_{11}^{0} \leq \lambda_{1}^{-}<\lambda_{3}^{+} \leq v_{32}^{0} .
$$

Hence, an inexpensive and guaranteed upper bound on $\lambda_{\max }$ is $\max \left(\left(v_{11}^{0}\right)_{-},\left(v_{32}^{0}\right)_{+}\right)$.

Remark 4.4. (Extra Newton step) Note that step 14 in Algorithm 1 is a Newton iteration. This step is optional, but we nevertheless include it to correct the bias introduced by the computation of $\tilde{p}^{*}$. Our experience is that $\tilde{p}^{*}$ is very often much closer to $p^{*}$ than both $p_{\min }$ and $p_{\max }$. The concavity of $\phi$ guaranties that $p_{2}^{0}-$ $\phi\left(p_{2}^{0}\right) / \phi^{\prime}\left(p_{2}^{0}\right)<p^{*}$, whence $p_{1}^{0}<p^{*}<p_{2}^{0}$ as desired.

Given two positive numbers $p_{1}, p_{2}$, we now construct two quadratic polynomials $P_{\mathrm{u}}(p)$ and $P_{\mathrm{d}}(p)$ such that $P_{\mathrm{u}}(p)$ interpolates $\phi$ at the points $p_{1}, p_{2}, p_{2}$ and $P_{\mathrm{d}}(p)$ 

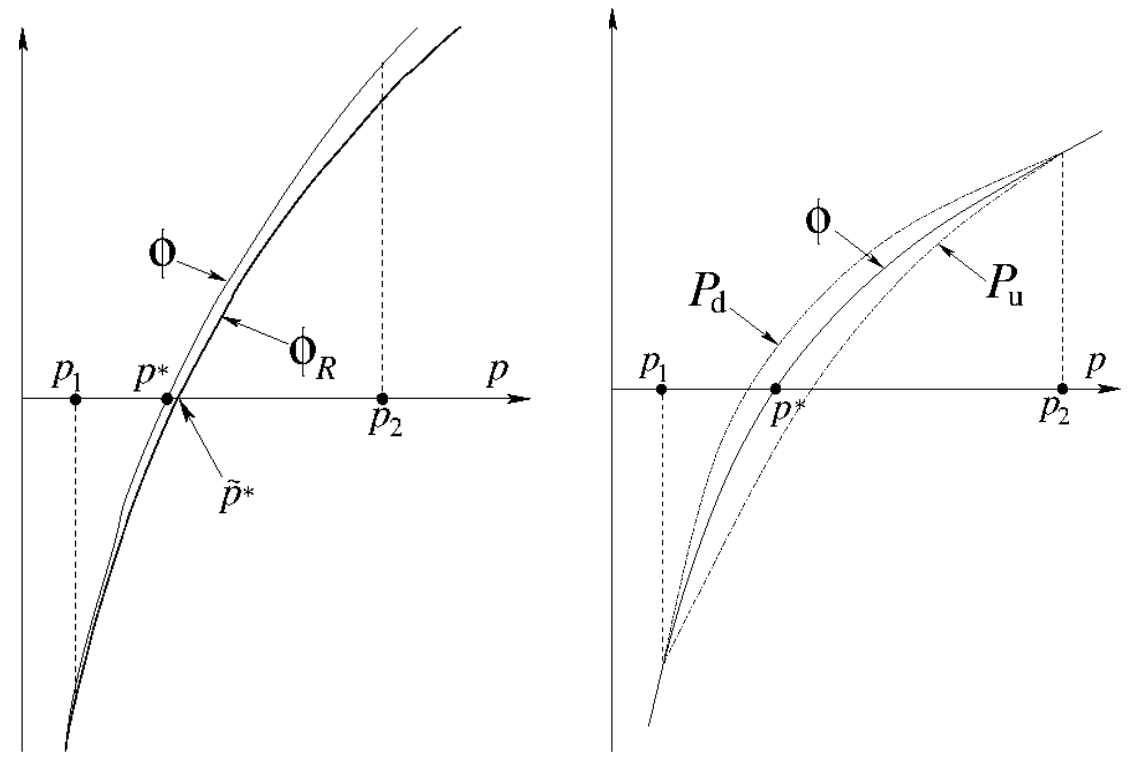

FIG. 4.1. Left: $\phi(p)=\phi_{R}(p)$ for all $p \leq p_{1}$ and $\phi_{R}(p)<\phi(p)$ for all $p \in\left(p_{\min }, \infty\right)$. Right: Quadratic polynomials $P_{u}(p)$ and $P_{d}(p)$.

interpolates $\phi$ at the points $p_{1}, p_{1}, p_{2}$.

$$
\begin{aligned}
& P_{\mathrm{d}}(p):=\phi\left(p_{1}\right)+\phi\left[p_{1}, p_{1}\right]\left(p-p_{1}\right)+\phi\left[p_{1}, p_{1}, p_{2}\right]\left(p-p_{1}\right)^{2}, \\
& P_{\mathrm{u}}(p):=\phi\left(p_{2}\right)+\phi\left[p_{2}, p_{2}\right]\left(p-p_{2}\right)+\phi\left[p_{1}, p_{2}, p_{2}\right]\left(p-p_{2}\right)^{2} .
\end{aligned}
$$

Here we use the standard divided difference notation where repeating a point means that we interpolate the function and its derivative at the said point. We abuse the notation by omitting the index $k$ for the two polynomials $P_{\mathrm{u}}$ and $P_{\mathrm{d}}$.

Lemma 4.5. $P_{\mathrm{u}}$ and $P_{\mathrm{d}}$ have each a unique zero over the interval $\left(p_{1}, p_{2}\right)$ denoted $p_{\mathrm{u}}\left(p_{1}, p_{2}\right)$ and $p_{\mathrm{d}}\left(p_{1}, p_{2}\right)$, respectively:

$$
\begin{aligned}
& p_{\mathrm{d}}\left(p_{1}, p_{2}\right)=p_{1}-\frac{2 \phi\left(p_{1}\right)}{\phi^{\prime}\left(p_{1}\right)+\sqrt{\phi^{\prime}\left(p_{1}\right)^{2}-4 \phi\left(p_{1}\right) \phi\left[p_{1}, p_{1}, p_{2}\right]}} \\
& p_{\mathrm{u}}\left(p_{1}, p_{2}\right)=p_{2}-\frac{2 \phi\left(p_{2}\right)}{\phi^{\prime}\left(p_{2}\right)+\sqrt{\phi^{\prime}\left(p_{2}\right)^{2}-4 \phi\left(p_{2}\right) \phi\left[p_{1}, p_{2}, p_{2}\right]}} .
\end{aligned}
$$

and the following holds for any $p \in\left(p_{1}, p_{2}\right)$ :

$$
P_{\mathrm{u}}(p)<\phi(p)<P_{\mathrm{d}}(p), \quad \forall p \in\left(p_{1}, p_{2}\right)
$$

which implies that $p_{1}<p_{\mathrm{d}}\left(p_{1}, p_{2}\right)<p^{*}<p_{\mathrm{u}}\left(p_{1}, p_{2}\right)<p_{2}$.

Proof. It is a standard result in approximation theory that

$$
\begin{aligned}
& \phi(p)-P_{\mathrm{u}}(p)=\phi\left[p_{1}, p_{2}, p_{2}, p\right]\left(p-p_{1}\right)\left(p-p_{2}\right)^{2} \\
& \phi(p)-P_{\mathrm{d}}(p)=\phi\left[p_{1}, p_{1}, p_{2}, p\right]\left(p-p_{1}\right)^{2}\left(p-p_{2}\right)
\end{aligned}
$$

where $\phi\left[p_{1}, p_{2}, p_{2}, p\right]$ and $\phi\left[p_{1}, p_{1}, p_{2}, p\right]$ are divided differences. For completeness we recall that $f[x]=f(x)$ and given $x_{0} \leq \ldots \leq x_{n}$ we have $f\left[x_{0}, \ldots, x_{n}\right]=\frac{1}{n !} f^{(n)}\left(x_{0}\right)$ 
if $x_{0}=\ldots=x_{n}$ and $f\left[x_{0}, \ldots, x_{n}\right]=\frac{f\left[x_{0}, \ldots, x_{n-1}\right]-f\left[x_{1}, \ldots, x_{n}\right]}{x_{0}-x_{n}}$ otherwise. Moreover we define $f\left[x_{\sigma(0)}, \ldots, x_{\sigma(n)}\right]=f\left[x_{0}, \ldots, x_{n}\right]$ for any $\sigma \in \mathcal{S}^{n+1}$ where $\mathcal{S}^{n+1}$ is the set all the permutations over the set $\{0, \ldots, n\}$. It is known that for any $x_{0}, \ldots, x_{n}$ we have $f\left[x_{0}, \ldots, x_{n}\right]=\frac{1}{n !} f^{(n)}(\xi)$ for some $\xi \in\left[\min \left(x_{0}, \ldots, x_{n}\right), \max \left(x_{0}, \ldots, x_{n}\right)\right]$. In the case at hand, we know that $\phi^{\prime \prime \prime}(\xi)>0$ for any $\xi>0$, then (4.10) is a simple consequence of (4.11). Both quadratic polynomials $P_{\mathrm{u}}$ and $P_{\mathrm{d}}$ are concave down, and both are negative at $p=p_{1}$ and positive at $p=p_{2}$; hence they each have a unique zero in the interval $\left(p_{1}, p_{2}\right)$, which we denote $p_{\mathrm{u}}\left(p_{1}, p_{2}\right)$ and $p_{\mathrm{d}}\left(p_{1}, p_{2}\right)$ respectively. Moreover, the inequality (4.10) implies that $p_{\mathrm{d}}\left(p_{1}, p_{2}\right)<p^{*}<p_{\mathrm{u}}\left(p_{1}, p_{2}\right)$. The proof is complete.

The result of Lemma 4.5 is illustrated in the right panel of Figure 4.1. The algorithm that we propose proceeds as follows: given a pair $\left(p_{1}^{k}, p_{2}^{k}\right)$, compute $\left(p_{1}^{k+1}, p_{2}^{k+1}\right)$ such that $p_{1}^{k+1}=p_{\mathrm{d}}\left(p_{1}^{k}, p_{2}^{k}\right)$ and $p_{2}^{k+1}=p_{\mathrm{u}}\left(p_{1}^{k}, p_{2}^{k}\right)$ for $k \geq 0$. Owing to Lemma 4.5, we have $p_{1}^{k} \leq p_{1}^{k+1} \leq p^{*} \leq p_{2}^{k+1} \leq p_{2}^{k}$ and the convergence rate of the iteration process is cubic.

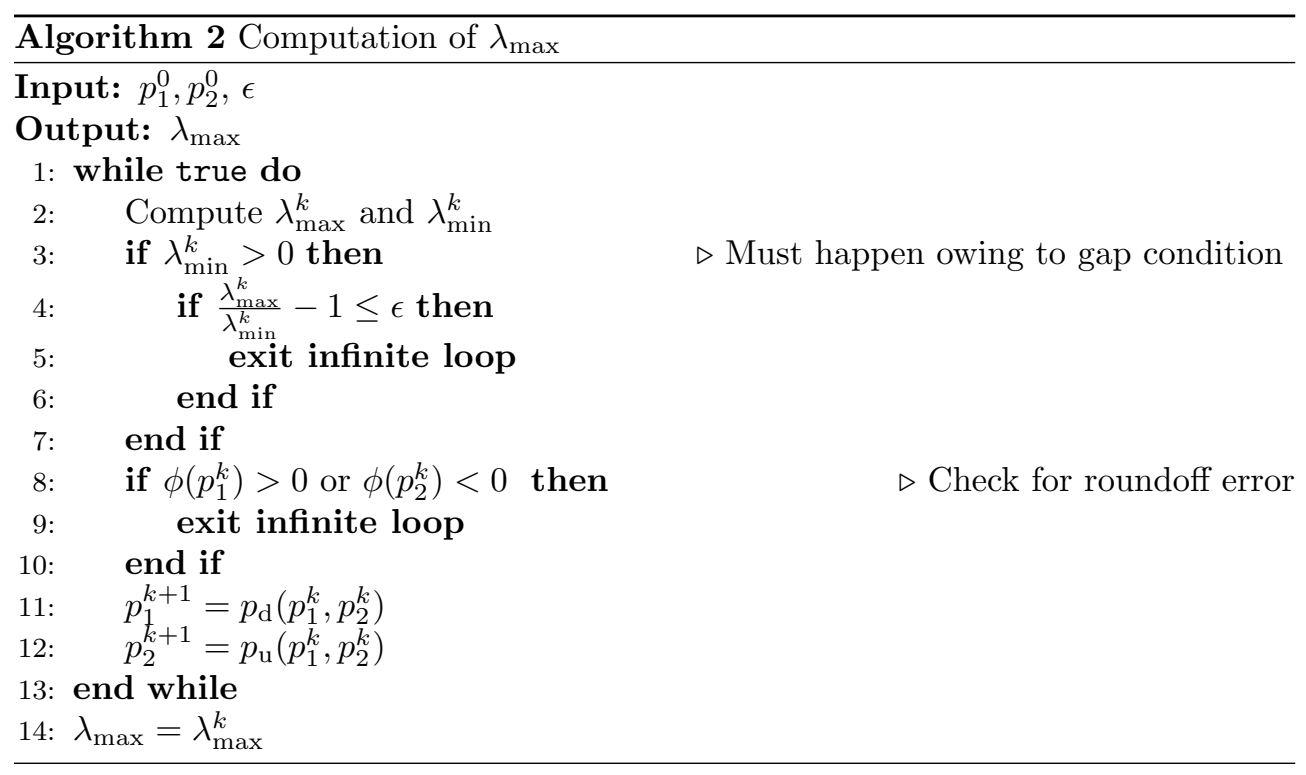

THEOREM 4.6. For every $\epsilon>0$, there exists $k\left(u_{L}, a_{L}, u_{R}, a_{R}, \gamma, \epsilon\right)$ such that Algorithm 2 terminates when $k=k\left(u_{L}, a_{L}, u_{R}, a_{R}, \gamma, \epsilon\right)$, and in this case

$$
\left|\lambda_{\max }^{k}-\lambda_{\max }\right| \leq \epsilon \lambda_{\max }
$$

i.e., the relative error on $\lambda_{\max }$ is guaranteed to be bounded by $\epsilon$.

Proof. Owing the gap Lemma 4.8, there is $c(\gamma)>0$ such that $\lambda_{3}^{+}-\lambda_{1}^{-} \geq$ $c(\gamma)\left(a_{\max }+a_{\min }\right)$, which in turn implies that $d:=\lambda_{3}^{+}-\lambda_{1}^{-}>0$ owing to the hyperbolicity condition $\min \left(a_{\max }, a_{\min }\right)>0$. Hence $\lambda_{\max }=\max \left(\left(\lambda_{3}^{+}\right)_{+},\left(\lambda_{1}^{-}\right)_{-}\right) \geq \frac{d}{2}>0$, (recall that $\left.\min \left(x_{-}, y_{+}\right) \geq \frac{|x-y|}{2}\right)$. Since both sequences $\left(p_{1}^{k}\right)_{k \geq 0}$ and $\left(p_{2}^{k}\right)_{k \geq 0}$ converge to $p^{*}$, there is $k_{0}\left(u_{L}, a_{L}, u_{R}, a_{R}, \gamma, \epsilon\right)$ such that $\lambda_{\min }^{k}>\frac{1}{2} \lambda_{\max } \geq \frac{1}{4} d>0$ for any $k \geq k_{0}\left(u_{L}, a_{L}, u_{R}, a_{R}, \gamma, \epsilon\right)$. Hence the condition of the if statement in line 3 of Algorithm 2 is achieved for any $k \geq k_{0}\left(u_{L}, a_{L}, u_{R}, a_{R}, \gamma, \epsilon\right)$. Likewise there is $k\left(u_{L}, a_{L}, u_{R}, a_{R}, \gamma, \epsilon\right) \geq k_{0}\left(u_{L}, a_{L}, u_{R}, a_{R}, \gamma, \epsilon\right)$ such that the condition of the if statement in line 4 holds true since $\lim _{k \rightarrow+\infty} \frac{\lambda_{\max }^{k}}{\lambda_{\min }^{k}}=1$. Hence, when $k=$ 
$k\left(u_{L}, a_{L}, u_{R}, a_{R}, \gamma, \epsilon\right)$ the algorithm terminates and the estimate (4.12) is guaranteed by Lemma 4.4. This completes the proof.

Remark 4.5. (Jacobi vs. Seidel iterations) Note that steps 11 and 12 in Algorithm 2 are of Jacobi type. The Seidel version of this algorithm is $p_{1}^{k+1}=p_{\mathrm{d}}\left(p_{1}^{k}, p_{2}^{k}\right)$, $p_{2}^{k+1}=p_{\mathrm{u}}\left(p_{1}^{k+1}, p_{2}^{k}\right)$.

Remark 4.6. (Roundoff errors) The algorithm converges so fast and is so accurate that it may happen that either the test $\phi\left(p_{1}^{k}\right)>0$ or the test $\phi\left(p_{2}^{k}\right)<0$ turns out to be true due to rounding errors. This then causes the discriminant in (4.9b) to be negative thereby producing NaN. To avoid the roundoff problem one must check the sign of $\phi\left(p_{1}^{k}\right)$ and $\phi\left(p_{2}^{k}\right)$ before computing $p_{1}^{k+1}$ and $p_{2}^{k+1}$ (see line 8 in Algorithm 2). If $\phi\left(p_{1}^{k}\right)>0$ then $p^{*}=p_{1}^{k}$ up to roundoff errors and if $\phi\left(p_{2}^{k}\right)<0$ then $p^{*}=p_{2}^{k}$ up to roundoff errors.

4.3. Estimation of $\lambda_{1}^{-}$and $\lambda_{3}^{+}$. In some applications it may be important to estimate both the left and the right wave speeds $\lambda_{1}^{-}$and $\lambda_{3}^{+}$. This is the case for instance in the so-called central-upwind schemes, see e.g., Kurganov et al. [17, §3.1], in the HLL, HLLC, HLLE approximate Riemann solvers, see Godlewski and Raviart [10, p. 204, Rem 3.2], and in Arbitrary Lagrangian Eleurian hydrodynamics, see e.g., Guermond et al. [12]. It is possible to modify the Algorithm 2 for this purpose as stated in the following corollary.

Corollary 4.7 (Bounds on $\lambda_{1}^{-}, \lambda_{3}^{+}$). Consider Algorithm 2 with line $4 \mathrm{re}$ placed by "4: if $\left(\frac{v_{12}^{k}-v_{11}^{k}}{\lambda_{\min }^{k}} \leq \epsilon\right.$ and $\left.\frac{v_{32}^{k}-v_{31}^{k}}{\lambda_{\min }^{k}} \leq \epsilon\right)$ then" and line 14 changed to return $\left(v_{11}^{k}, v_{32}^{k}\right)$. Then for every $\epsilon>0$, there exists an integer $k\left(u_{L}, a_{L}, u_{R}, a_{R}, \gamma, \epsilon\right)$ such that Algorithm 2 terminates when $k=k\left(u_{L}, a_{L}, u_{R}, a_{R}, \gamma, \epsilon\right)$, and in this case $v_{11}^{k}$ and $v_{32}^{k}$ satisfy the following guaranteed bounds:

$$
0 \leq \lambda_{1}^{-}-v_{11}^{k} \leq \epsilon \lambda_{\max }, \quad 0 \leq v_{32}^{k}-\lambda_{3}^{+} \leq \epsilon \lambda_{\max },
$$

i.e., the error on $\lambda_{1}^{-}$and $\lambda_{3}^{+}$relative to $\lambda_{\max }$ is guaranteed to be bounded by $\epsilon$.

Note that in the above corollary $\lambda_{1}^{-}$is approximated from below and $\lambda_{3}^{+}$is approximated from above. Hence, we always guarantee that the exact Riemann fan is contained in the cone $C^{k}:=\left\{(x, t) \mid v_{11}^{k} t \leq x \leq v_{32}^{k} t, 0<t\right\}$ for any $k \geq 0$. Note also that $C^{k+1} \subset C^{k}$ for any $k \geq 0$.

4.4. Gap condition. The purpose of this section is to establish the following result, which we call the gap condition.

LEMMA 4.8 (Gap condition). Given the left state $\boldsymbol{c}_{L}:=\left(\rho_{L}, m_{L}, \mathcal{E}_{L}\right)$ and the right state $\boldsymbol{c}_{R}:=\left(\rho_{R}, m_{R}, \mathcal{E}_{R}\right)$ of the one-dimensional Riemann problem (2.6), we have the following gap condition for the smallest and largest eigenvalues of the problem

$$
\lambda_{3}^{+}-\lambda_{1}^{-} \geq c(\gamma)\left(a_{L}+a_{R}\right)
$$

where $a_{L}, a_{R}$ are the local sound speeds and $c(\gamma)$ is a constant defined by

$$
c(\gamma):= \begin{cases}\frac{2 \sqrt{2(\gamma-1)}}{\gamma+1} & \text { if } \gamma \in(1,3], \\ 1 & \text { if } \gamma \in(3,+\infty) .\end{cases}
$$

Proof. There are three possible cases for the solution of the Riemann problem.

Case 1. The solution contains two rarefaction waves: $\phi\left(p_{\min }\right) \geq 0$. This implies that either there exists $p^{*} \geq 0$ such that $\phi\left(p^{*}\right)=0$ or we have vacuum, i.e., $0 \leq \phi(0)$. 
If $\phi\left(p^{*}\right)=0$ we derive

$$
u_{R}-u_{L}=\frac{2 a_{L}\left(1-b \rho_{L}\right)}{\gamma-1}\left(1-\left(\frac{p^{*}}{p_{L}}\right)^{\frac{\gamma-1}{2 \gamma}}\right)+\frac{2 a_{R}\left(1-b \rho_{R}\right)}{\gamma-1}\left(1-\left(\frac{p^{*}}{p_{R}}\right)^{\frac{\gamma-1}{2 \gamma}}\right) \geq 0,
$$

and in the case of vacuum we get

$$
u_{R}-u_{L} \geq \frac{2}{\gamma-1}\left(a_{L}\left(1-b \rho_{L}\right)+a_{R}\left(1-b \rho_{R}\right)\right)>0 .
$$

Using the fact that $p^{*} \leq p_{\min }$, we derive from (3.7)-(3.8) that

$$
\lambda_{3}^{+}-\lambda_{1}^{-}=u_{R}-u_{L}+a_{L}+a_{R} \geq a_{L}+a_{R},
$$

which proves (4.14) with constant $c(\gamma)=1$ in this case.

Case 2. The solution contains one rarefaction and one shock wave: $\phi\left(p_{\min }\right)<0=$ $\phi\left(p^{*}\right) \leq \phi\left(p_{\max }\right)$. Then, we have that $p_{\min }<p^{*} \leq p_{\max }$ and

$0=\phi\left(p^{*}\right)=\left(p^{*}-p_{\min }\right) \sqrt{\frac{A_{\min }}{p^{*}+B_{\min }}}+\frac{2 a_{\max }\left(1-b \rho_{\max }\right)}{\gamma-1}\left(\left(\frac{p^{*}}{p_{\max }}\right)^{\frac{\gamma-1}{2 \gamma}}-1\right)+u_{R}-u_{L}$

where we recall that $A_{Z}:=\frac{2\left(1-b \rho_{Z}\right)}{(\gamma+1) \rho_{Z}}$ and $B_{Z}:=\frac{\gamma-1}{\gamma+1} p_{Z}$. Using the above we derive the following

$$
\begin{aligned}
\lambda_{3}^{+}-\lambda_{1}^{-} & =a_{\max }\left(1+\frac{2\left(1-b \rho_{\max }\right)}{\gamma-1}\left(1-\left(\frac{p^{*}}{p_{\max }}\right)^{\frac{\gamma-1}{2 \gamma}}\right)\right) \\
& +a_{\min }\left(\left(1+\frac{\gamma+1}{2 \gamma} \frac{p^{*}-p_{\min }}{p_{\min }}\right)^{\frac{1}{2}}-\frac{\left(p^{*}-p_{\min }\right)}{a_{\min }} \sqrt{\frac{A_{\min }}{p^{*}+B_{\min }}}\right) \\
& \geq a_{\max }+a_{\min } \Delta\left(p_{\min }\right) .
\end{aligned}
$$

where we define $\Delta(Z)$ by

$$
\Delta(Z)=\left(1+\frac{\gamma+1}{2 \gamma} \frac{p^{*}-p_{Z}}{p_{Z}}\right)^{\frac{1}{2}}-\frac{p^{*}-p_{Z}}{a_{Z}} \sqrt{\frac{A_{Z}}{p^{*}+B_{Z}}} .
$$

We make a substitution $x=\frac{p^{*}}{p_{\min }} \geq 1$ and, with a an abuse of notation, we transform $\Delta\left(p_{\min }\right)$ into

$$
\Delta(x)=\left(1+\frac{\gamma+1}{2 \gamma}(x-1)\right)^{\frac{1}{2}}-\frac{(x-1)\left(1-b \rho_{\min }\right)}{\left(x+\frac{\gamma-1}{\gamma+1}\right)^{\frac{1}{2}}} \sqrt{\frac{2}{\gamma(\gamma+1)}} .
$$

Using the property that $0 \leq 1-b \rho_{\min } \leq 1$, we derive

$$
\Delta(x) \geq \Delta_{0}(x):=\left(1+\frac{\gamma+1}{2 \gamma}(x-1)\right)^{\frac{1}{2}}-\frac{x-1}{\left(x+\frac{\gamma-1}{\gamma+1}\right)^{\frac{1}{2}}} \sqrt{\frac{2}{\gamma(\gamma+1)}} .
$$

We now want to find the minimum of the function $\Delta_{0}(x)$ in the interval $x \in[1,+\infty)$. We transform $\Delta_{0}(x)$ as follows

$$
\Delta_{0}(x)=\left(\frac{\gamma+1}{2 \gamma}\right)^{\frac{1}{2}}\left(x+\frac{\gamma-1}{\gamma+1}\right)^{-\frac{1}{2}}\left(x+\frac{\gamma-1}{\gamma+1}-(x-1) \frac{2}{\gamma+1}\right)
$$


and after another substitution $y=x-1$, and another abuse of notation, we have

$$
\Delta_{0}(y)=\left(\frac{\gamma+1}{2 \gamma}\right)^{\frac{1}{2}}\left(y+\frac{2 \gamma}{\gamma+1}\right)^{-\frac{1}{2}}\left(\frac{\gamma-1}{\gamma+1} y+\frac{2 \gamma}{\gamma+1}\right)=\frac{\gamma-1}{(2 \gamma(\gamma+1))^{\frac{1}{2}}} \psi(y)
$$

where $\psi(y)=\left(y+\frac{2 \gamma}{\gamma-1}\right)\left(y+\frac{2 \gamma}{\gamma+1}\right)^{-\frac{1}{2}}$. The function $\psi(y)$ has a unique minimum on the interval $[0,+\infty)$ at the point $y_{\min }=\frac{2 \gamma(3-\gamma)}{(\gamma-1)(\gamma+1)}$ provided that $\gamma \leq 3$. The value of the minimum is $\psi\left(y_{\min }\right)=4 \sqrt{\frac{\gamma}{(\gamma-1)(\gamma+1)}}$. If $\gamma>3$ then the minimum is at $y=0$ and the value is $\psi(0)=\frac{\sqrt{(\gamma+1)}}{\gamma-1}$. Using the minimum value of $\psi$ we get the following minimum of the function $\Delta_{0}(x)$ on the interval $[1,+\infty)$ :

$$
\Delta_{0}(x) \geq \frac{2 \sqrt{2(\gamma-1)}}{\gamma+1} \text { if } \gamma \in(1,3], \quad \text { and } \quad \Delta_{0}(x) \geq 1 \text { if } \gamma \in(3,+\infty) .
$$

This finishes the proof in the second case because for the full range of $\gamma$ we get $\Delta(x) \geq \Delta_{0}(x) \geq c(\gamma)$. This again proves (4.14) since $c(\gamma) \leq 1$ for any $\gamma \in[1, \infty)$.

Case 3. The solution contains two shock waves: $\phi\left(p_{\min }\right) \leq \phi\left(p_{\max }\right)<0=\phi\left(p^{*}\right)$. Then, we have that $p_{\min } \leq p_{\max }<p^{*}$ and

$$
0=\phi\left(p^{*}\right)=\left(p^{*}-p_{\min }\right) \sqrt{\frac{A_{\min }}{p^{*}+B_{\min }}}+\left(p^{*}-p_{\max }\right) \sqrt{\frac{A_{\max }}{p^{*}+B_{\max }}}+u_{R}-u_{L}
$$

Similar to the previous case we derive

$$
\begin{aligned}
\lambda_{3}^{+}-\lambda_{1}^{-} & =a_{\min }\left(\left(1+\frac{\gamma+1}{2 \gamma} \frac{p^{*}-p_{\min }}{p_{\min }}\right)^{\frac{1}{2}}-\frac{\left(p^{*}-p_{\min }\right)}{a_{\min }} \sqrt{\frac{A_{\min }}{p^{*}+B_{\min }}}\right) \\
& +a_{\max }\left(\left(1+\frac{\gamma+1}{2 \gamma} \frac{p^{*}-p_{\max }}{p_{\max }}\right)^{\frac{1}{2}}-\frac{\left(p^{*}-p_{\max }\right)}{a_{\max }} \sqrt{\frac{A_{\max }}{p^{*}+B_{\max }}}\right) \\
& \geq a_{\max } \Delta\left(p_{\min }\right)+a_{\min } \Delta\left(p_{\min }\right)
\end{aligned}
$$

where $\Delta(Z)$ is the same as before, see (4.4). We now use the fact that $\Delta(Z) \geq c(\gamma)$ when $p_{Z} \leq p^{*}$ to finish the proof in this case.

5. Numerical illustrations. We illustrate the performances of Algorithm 2 in this section. We only consider test cases where there is at least one shock, since the cases with two expansion waves are trivial. The set of test problems we use is based on the performance tests given in Toro [20, Section 4.3.3]. The code that we used is included in Appendix C.

5.1. Fast expansion and slow shock. Algorithm 2 may terminate and give an estimate on $\lambda_{\max }$ with the required accuracy before $p^{*}$ is estimated correctly. This situation may happen when one of the two extreme waves is a fast expansion (rarefaction) and the other wave is a slow shock. To illustrate this effect, let us assume for instance that the left wave is a fast expansion and the right wave is the slow shock, say $p_{L}>p^{*}>p_{R}$ and $\left(\lambda_{1}^{-}\right)_{-}>\left(\lambda_{3}^{-}\right)_{+}=\left(\lambda_{3}^{+}\right)_{+}$. Note that in this case we always have $p_{R} \leq p_{1}^{k} \leq p_{2}^{k} \leq p_{L}$ for any $k \geq 0$; hence, $v_{11}^{k}=v_{12}^{k}=u_{L}-a_{L}=\lambda_{1}^{-}$for any $k \geq 0$. At some point in the algorithm there will be an iteration level $k$ such that 
both $p_{1}^{k}$ and $p_{2}^{k}$ are close enough to $p^{*}$ so that $0 \leq v_{32}^{k}-\lambda_{3}^{+} \leq\left(\lambda_{1}^{-}\right)_{-}-\left(\lambda_{3}^{+}\right)_{+}$and $0 \leq v_{31}^{k}-\lambda_{3}^{+} \leq\left(\lambda_{1}^{-}\right)_{-}-\left(\lambda_{3}^{+}\right)_{+}$. Hence, using that $x \geq y$ implies that $x-y+y_{+}-x_{+} \geq 0$, we have

$$
\begin{aligned}
\left(v_{11}^{k}\right)_{-}-\left(v_{32}^{k}\right)_{+} & =\left(\lambda_{1}^{-}\right)_{-}-v_{32}^{k}+v_{32}^{k}-\left(v_{32}^{k}\right)_{+} \\
& \geq\left(\lambda_{3}^{+}\right)_{+}-\lambda_{3}^{+}+v_{32}^{k}-\left(v_{32}^{k}\right)_{+} \geq 0 .
\end{aligned}
$$

This means that $\lambda_{\max }^{k}:=\max \left(\left(v_{32}^{k}\right)_{+},\left(v_{11}^{k}\right)_{-}\right)=\left(v_{11}^{k}\right)_{-}=\left(\lambda_{1}^{-}\right)_{-}$. Likewise

$$
\begin{aligned}
\left(v_{12}^{k}\right)_{-}-\left(v_{31}^{k}\right)_{+} & =\left(\lambda_{1}^{-}\right)_{-}-v_{31}^{k}+v_{31}^{k}-\left(v_{31}^{k}\right)_{+} \\
& \geq\left(\lambda_{3}^{+}\right)_{+}-\lambda_{3}^{+}+v_{31}^{k}-\left(v_{31}^{k}\right)_{+} \geq 0,
\end{aligned}
$$

i.e., $\lambda_{\min }^{k}:=\max \left(\left(v_{31}^{k}\right)_{+},\left(v_{12}^{k}\right)_{-}\right)_{+}=\left(v_{12}^{k}\right)_{-}=\left(\lambda_{1}^{-}\right)_{-}$. In conclusion at iteration $k$, we have $\frac{\lambda_{\max }^{k}}{\lambda_{\min }^{k}}-1=0$; in other word the algorithm stops irrespective of the tolerance, and $\lambda_{\max }^{k}=\lambda_{1}^{-}$but $p_{1}^{k}$ and $p_{2}^{k}$ may still be far from $p^{*}$. To illustrate this phenomenon

\begin{tabular}{|c|c|c|c|c|c|c|c|}
\hline & & & & & & \\
\hline & & case & $k$ & \multicolumn{2}{|c|}{$\lambda_{\max }^{k}$} & \multicolumn{2}{|c|}{$\lambda_{\max }$} \\
\hline & & 1 & 0 & \multicolumn{2}{|c|}{11.83215956619923} & \multicolumn{2}{|c|}{11.83215956619923} \\
\hline & & 2 & 1 & \multicolumn{2}{|c|}{10.83215956619923} & \multicolumn{2}{|c|}{10.83215956619923} \\
\hline & & 3 & 2 & \multicolumn{2}{|c|}{9.65215956619923} & \multicolumn{2}{|c|}{9.65215956619923} \\
\hline case & $k$ & \multicolumn{3}{|c|}{$p_{1}^{k}$} & \multicolumn{2}{|c|}{$p_{2}^{k}$} & $\overline{p^{*}}$ \\
\hline 1 & 0 & \multicolumn{3}{|c|}{37.70559999364363} & \multicolumn{2}{|c|}{82.98306927558072} & 46.09504424886797 \\
\hline 2 & 1 & \multicolumn{3}{|c|}{45.87266091833658} & \multicolumn{2}{|c|}{46.70007404915459} & 46.09504424886797 \\
\hline 3 & 2 & \multicolumn{3}{|c|}{46.09504109404150} & \multicolumn{2}{|c|}{46.09505272562230} & 46.09504424886797 \\
\hline
\end{tabular}
we consider the following test cases:

\begin{tabular}{||c|c|c|c|c|c|c||}
\hline case & $\rho_{L}$ & $\rho_{R}$ & $u_{L}$ & $u_{R}$ & $p_{L}$ & $p_{R}$ \\
\hline 1 & 1.0 & 1.0 & 0.0 & 0.0 & 0.01 & 100.0 \\
\hline 2 & 1.0 & 1.0 & -1.0 & -1.0 & 0.01 & 100.0 \\
\hline 3 & 1.0 & 1.0 & -2.18 & -2.18 & 0.01 & 100.0 \\
\hline
\end{tabular}

We run the algorithm with $\epsilon=10^{-15}$. The results are

In the first case, The algorithm terminates just after the initialization, i.e., at $k=$ 0 , and gives the exact value of $\lambda_{\max }$ up to a rounding error, but it gives $p_{1}^{0}<p^{*}<p_{2}^{0}$. In the second case the algorithm terminates at $k=1$ with the exact value of $\lambda_{\max }$ up to a rounding error, but it gives $p_{1}^{1}<p^{*}<p_{2}^{1}$. By biasing the problem a little bit more to the right, i.e., by taking $u_{L}=u_{R}=2.18$, the algorithm terminates at $k=2$ and gives again the exact value of $\lambda_{\max }$ up to a rounding error, but $p_{1}^{2}<p^{*}<p_{2}^{2}$. We have verified that for $u_{L}=u_{R} \geq 2.2$ the right-moving shock wave is the fastest and the algorithm always terminates at $k=3$ and gives $p_{1}^{k}=p^{*}=p_{2}^{k}$ up to round-off errors.

5.2. Fast shock. The most demanding situation happens when the fastest wave is a shock, since in this case the algorithm must find $p^{*}$ up to the assigned tolerance to terminate. We consider the following two cases introduced in Toro [20, Section 4.3.3]:

\begin{tabular}{||c|c|c|c|c|c|c||}
\hline case & $\rho_{L}$ & $\rho_{R}$ & $u_{L}$ & $u_{R}$ & $p_{L}$ & $p_{R}$ \\
\hline 1 & 1.0 & 1.0 & 10.0 & 10.0 & 1000.0 & 0.01 \\
\hline 2 & 5.99924 & 5.99242 & 19.5975 & -6.19633 & 460.894 & 46.0950 \\
\hline
\end{tabular}


In case 1 , the left wave is a rarefaction and the right wave is a shock. In case 2 , both waves are shocks. We run the algorithm with various values of $\epsilon$; the results are

\begin{tabular}{||c|c|c|c|c|c||}
\hline & $\epsilon$ & $k$ & $\lambda_{\max }^{k}$ & $p_{1}^{k}$ & $p_{2}^{k}$ \\
\hline 1 & $10^{-1}$ & 1 & 33.81930602421521 & 455.2466713625296 & 472.7977828960125 \\
\hline 1 & $10^{-2}$ & 2 & 33.51755796979217 & 460.8933865271423 & 460.8946107187795 \\
\hline 1 & $10^{-15}$ & 3 & 33.51753696690324 & 460.8937874913834 & 460.8937874913835 \\
\hline \hline 2 & $10^{-1}$ & 1 & 12.25636731290528 & 1691.520678281327 & 1692.676852734373 \\
\hline 2 & $10^{-4}$ & 2 & 12.25077812313116 & 1691.646955398068 & 1691.646955407751 \\
\hline 2 & $10^{-15}$ & 3 & 12.25077812308434 & 1691.646955399126 & 1691.646955399126 \\
\hline
\end{tabular}

We observe that the algorithm converges very fast and it takes three steps to reach $10^{-15}$ accuracy on $\lambda_{\max }$. These two examples are representative of all the tests we have done in that most of the times the tolerance $10^{-15}$ is achieved in at most three steps.

When running the code $1,000,000$ times on case 2 with $10^{-15}$ tolerance, which amounts to three iterations per case, the total CPU time was 0.736 seconds on a machine with the following characteristics: Intel(R) Xeon(R) CPU E3-1220 v3 3.10GHz.

5.3. Overhead estimation. In order to give an idea of the overhead cost of estimating the maximum wave speed with the above algorithm, we test the code on the so-called Sod shocktube and Leblanc shocktube. The data for these two Riemann problems are recalled in the following table:

\begin{tabular}{||c|c|c|c|c|c|c|c||}
\hline case & $\gamma$ & $\rho_{L}$ & $\rho_{R}$ & $u_{L}$ & $u_{R}$ & $p_{L}$ & $p_{R}$ \\
\hline Sod & $7 / 5$ & 1.0 & 0.125 & 0.0 & 0.0 & 1.0 & 0.1 \\
\hline Leblanc & $5 / 3$ & 1.0 & 0.001 & 0.0 & 0.0 & 0.1 & $10^{-10}$ \\
\hline
\end{tabular}

The problems are set in two space dimensions in the domain $(0,0.1) \times(0,1)$ and are solved by using a finite element technique described in Guermond and Popov [11]. It is an explicit method using continuous finite elements on unstructured grids that works in any space dimension. The time stepping is done by using the $\operatorname{SPP} \operatorname{RK}(3,3)$ algorithm. The two-dimensional triangular mesh that is used for this test is shown on the top panel of Figure 5.1. The mesh is composed of 2400 triangles and 1311 vertices. The stopping time and number of time sub-steps performed are $t=0.4$ and 1170 for the Leblanc test case, respectively, and $t=0.2$ and 1101 for the Sod test case, respectively. For each time sub-step we use the code whose source is given in $\S \mathrm{C}$ to estimate the maximum wave speed. This code is an exact transcription in Fortran 95 of Algorithms 1 and 2. The code is called two times for every edge in the mesh at every Runge Kutta substep. For each sub-step we record the number of times the maximum wave speed code is called, say $N_{\text {tot }}$, and the number of times the algorithm enters the iterative loop, say $N_{\text {iter }}$, i.e., the number of times the algorithm goes beyond the exit test in line 13 in Algorithm 1 times the number of iterations in the iterative loop. We call overhead the ratio $N_{\text {iter }} / N_{\text {tot }}$; note that this number is greater than or equal to 1 if one never exits at line 13 in Algorithm 1. We show in the left and right panels of Figure 5.1 the overhead as a function of the time index for various values of the tolerance $\epsilon$ (denoted "tol" in the figure keys). We have observed that the overhead is 0 for the Sod test for $\epsilon \geq 6 \times 10^{-3}$ (data not shown here). In both the Sod and Leblanc cases we observe that the overhead decreases rapidly after a few time sub-steps. The overhead is at most $1 \%$ for the Sod test at the beginning and is 


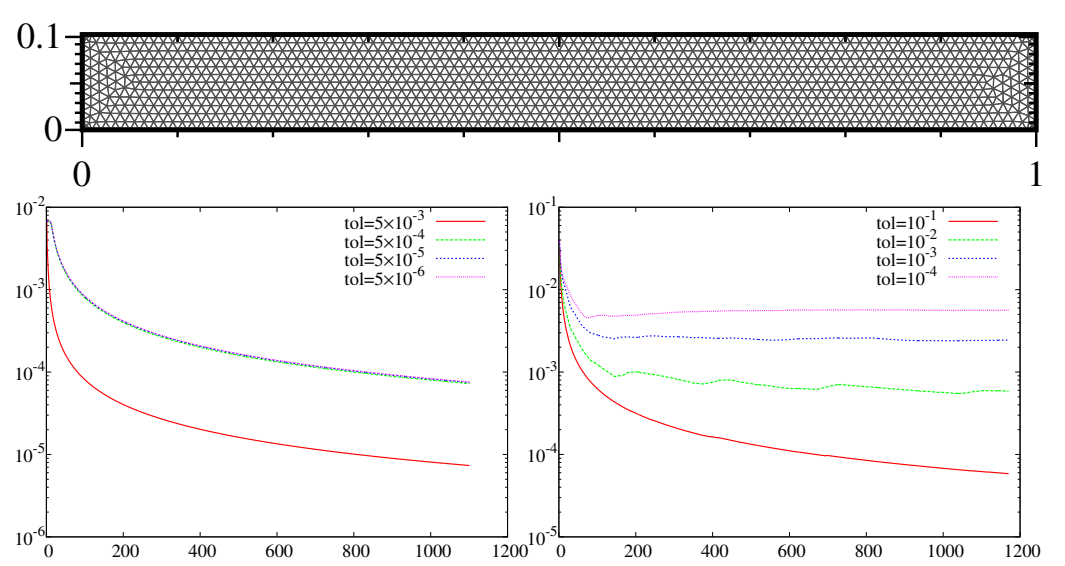

FIG. 5.1. Overhead $N_{\text {iter }} / N_{\text {tot }}$ vs. time iterations for the Sod shocktube (left panel) and the Leblanc shocktube (right panel) on the Delaunay mesh shown on the top panel.

less that $0.1 \%$ after 100 time sub-steps; the overhead does not to depend a lot on $\epsilon$ when $\epsilon \leq 5 \times 10^{-4}$. For the Leblanc test, the overhead is at most $10 \%$ at the beginning and is less than $1 \%$ after 10 substeps. There seems to be a weak dependency on $\epsilon$ in this test, but the overhead can be kept well below $1 \%$ for $\epsilon \leq 10^{-4}$.

Since for any practical purpose it is enough to take $\epsilon \in\left[10^{-3}, 5 \times 10^{-2}\right]$ to obtain an accurate estimate of the maximum wave speed in the algorithms described in [11, 12], the above tests show that the overhead of the proposed method is virtually less than $1 \%$.

Appendix A. Co-volume equation of state. We verify in this appendix the following statement.

Proposition A.1. If the left and right states in the Riemann problem (2.6) are such that $0<1-b \rho_{L}, 1-b \rho_{R}<1$, then the exact solution of the Riemann problem satisfies $0<1-b \rho \leq 1$.

Proof. We split the proof into five parts where we analyze the solution across each wave.

(1) Left rarefaction wave. Assume that the left wave is a rarefaction, then the wave speed, $S:=u-a=u-\sqrt{\frac{\gamma p}{\rho(1-b \rho)}}$, should increase along the wave from left to right. Using that both the specific entropy $s=\log \left(e^{\frac{1}{\gamma-1}}\left(\frac{1}{\rho}-b\right)\right)$ and the generalized left Riemann invariant $u+\frac{2 a}{\gamma-1}(1-b \rho)$ are constant across the left rarefaction, we obtain (see Eq. (4.93) and Eq. (4.94) in Toro [20, Chapter 4.7]):

$$
S(p)=u_{L}+\frac{2\left(1-b \rho_{L}\right)}{\gamma-1} a_{L}-\frac{2\left(1-b \rho+\frac{\gamma-1}{2}\right)}{(\gamma-1)(1-b \rho)} a_{L}\left(1-b \rho_{L}\right)\left(\frac{p}{p_{L}}\right)^{\frac{\gamma-1}{2 \gamma}}
$$

where $\frac{1}{\rho}-b=\left(\frac{1}{\rho_{L}}-b\right)\left(\frac{p_{L}}{p}\right)^{\frac{1}{\gamma}}$. Owing to the assumption $\frac{1}{\rho_{L}}-b>0$, we conclude that $\rho=\rho(p)$ is an increasing function of $p$. After some computation, we also prove that the speed $S(p)$ is a decreasing functions of $p$. Therefore the rarefaction wave can be parametrized by $p$ as a decreasing parameter from $p_{L}$ to $p^{*}$, i.e., the left wave is well defined. Hence we have $S\left(p_{L}\right) \leq S(p)$ for $p^{*}<p \leq p_{L}$, which confirms that we have a rarefaction wave. Finally, using that $\rho(p)$ is a decreasing function of $p$, we conclude 
that $0<1-b \rho_{L}<1-b \rho$. Observing also that $\rho=\rho_{L} \frac{1-b \rho_{L}}{1-b \rho}\left(\frac{p}{p_{L}}\right)^{\frac{1}{\gamma}}$ we conclude that $\rho \in\left[0, \rho_{L}\right]$ since $p^{*} \geq 0$ and $p \in\left[p^{*}, p_{L}\right]$.

(2) Left shock wave. Assume that the left wave is a shock from the left state $\left(\rho_{L}, u_{L}, p_{L}\right)$ to the state $(\rho, u, p)$. The Rankine-Hugoniot condition combined with the equation of state implies that (see e.g., Toro [20, Chapter 4.7])

$$
\rho=\frac{\rho_{L}\left(\frac{p}{p_{L}}+\frac{\gamma-1}{\gamma+1}\right)}{\frac{\gamma-1+2 b \rho_{L}}{\gamma+1} \frac{p}{p_{L}}+\frac{\gamma+1-2 b \rho_{L}}{\gamma+1}} .
$$

For details, we refer to Toro [20, (4.89) on p.145]. We introduce $y:=b \rho, y_{L}:=b \rho_{L}$ and $\beta:=\frac{p}{p_{L}}$ and the above equality can be re-written as follows:

$$
y=y_{L} \frac{\beta+\frac{\gamma-1}{\gamma+1}}{\frac{\gamma-1}{\gamma+1} \beta+1+\frac{2 y_{L}}{\gamma+1}(\beta-1)},
$$

which is equivalent to

$$
1-y=\frac{1}{1+\frac{2 y_{L}(\beta-1)}{(\gamma-1) \beta+\gamma+1}}\left(1-y_{L}\right) .
$$

Hence, we conclude that provided that $0 \leq y_{L}<1$ and $\beta \geq 1$, then $y_{L} \leq y<1$. This proves the result in the second case.

(3) Right rarefaction wave. The proof is analogous to the case of the left rarefaction wave.

(4) Right shock wave. The proof is analogous to the case of the left shock wave.

(5) Contact wave. The state on the left of the contact wave is the right state from the left wave which has already been proved to be admissible in 1-4. Similarly, the state on the right of the contact wave is the left state from the right wave which has also been proved to be admissible in 1-4. This completes the proof.

Appendix B. A counter-example. We show in this section that taking $\max \left(\left|u_{L}\right|+a_{L},\left|u_{R}\right|+a_{R}\right)$ as an estimate of the maximum wave speed in the Riemann problem, as it is frequently done in the literature, can actually underestimate the actual maximum wave speed.

For instance take $u_{L}=u_{R}=0$ and select $p_{L}$ and $p_{R}$ so that $p_{L} / p_{R}$ is a number less than 1. Then $a_{R}=a_{L} \sqrt{\frac{p_{R}}{p_{L}}} \sqrt{\frac{\rho_{L}}{\rho_{R}}}$. Now we choose $\frac{\rho_{L}}{\rho_{R}}$ so that $\sqrt{\frac{p_{R}}{p_{L}}} \sqrt{\frac{\rho_{L}}{\rho_{R}}}<1$; note that we can make this number as small as we want. Then $\max \left(\left|u_{L}\right|+a_{L},\left|u_{R}\right|+a_{R}\right)=a_{L}$. But $u_{L}=u_{R}=0$ and $p_{L}<p_{R}$ implies that $\phi\left(p_{L}\right)<0$ and $\phi\left(p_{R}\right)>0$; whence $p_{L}<p^{*}<p_{R}$. Therefore the maximum wave speed is the absolute value of the left speed given in $(3.7), a_{L}\left(1+\frac{\gamma+1}{2 \gamma}\left(\frac{p^{*}-p_{L}}{p_{L}}\right)\right)^{\frac{1}{2}}$, which is strictly larger than $a_{L}$, whence the conclusion.

To illustrate the above argument we now give two examples. First we consider case 2 from $\S 5.2$. The 1 -wave and the 3 -wave are both shocks. The correct wave speed is $\lambda_{\text {max }} \approx 12.25$ but the traditional estimate gives $\max \left(\left|u_{L}\right|+a_{L},\left|u_{R}\right|+a_{R}\right) \approx 29.97$, which is clearly an overestimate of $\lambda_{\max }$; the ratio is approximately 0.41. Second we consider the following two states

$$
\rho_{L}=0.01, \rho_{R}=1000, \quad u_{L}=0, u_{R}=0, \quad p_{L}=0.01, p_{R}=1000 .
$$


We obtain $\lambda_{\max } \approx 5.227$ and $\max \left(\left|u_{L}\right|+a_{L},\left|u_{R}\right|+a_{R}\right) \approx 1.183$. It is clear that the heuristic estimate is far from the real value; the ratio is approximately 4.4. In conclusion the estimate $\max \left(\left|u_{L}\right|+a_{L},\left|u_{R}\right|+a_{R}\right)$ is grossly unreliable.

\section{Appendix C. Source code.}

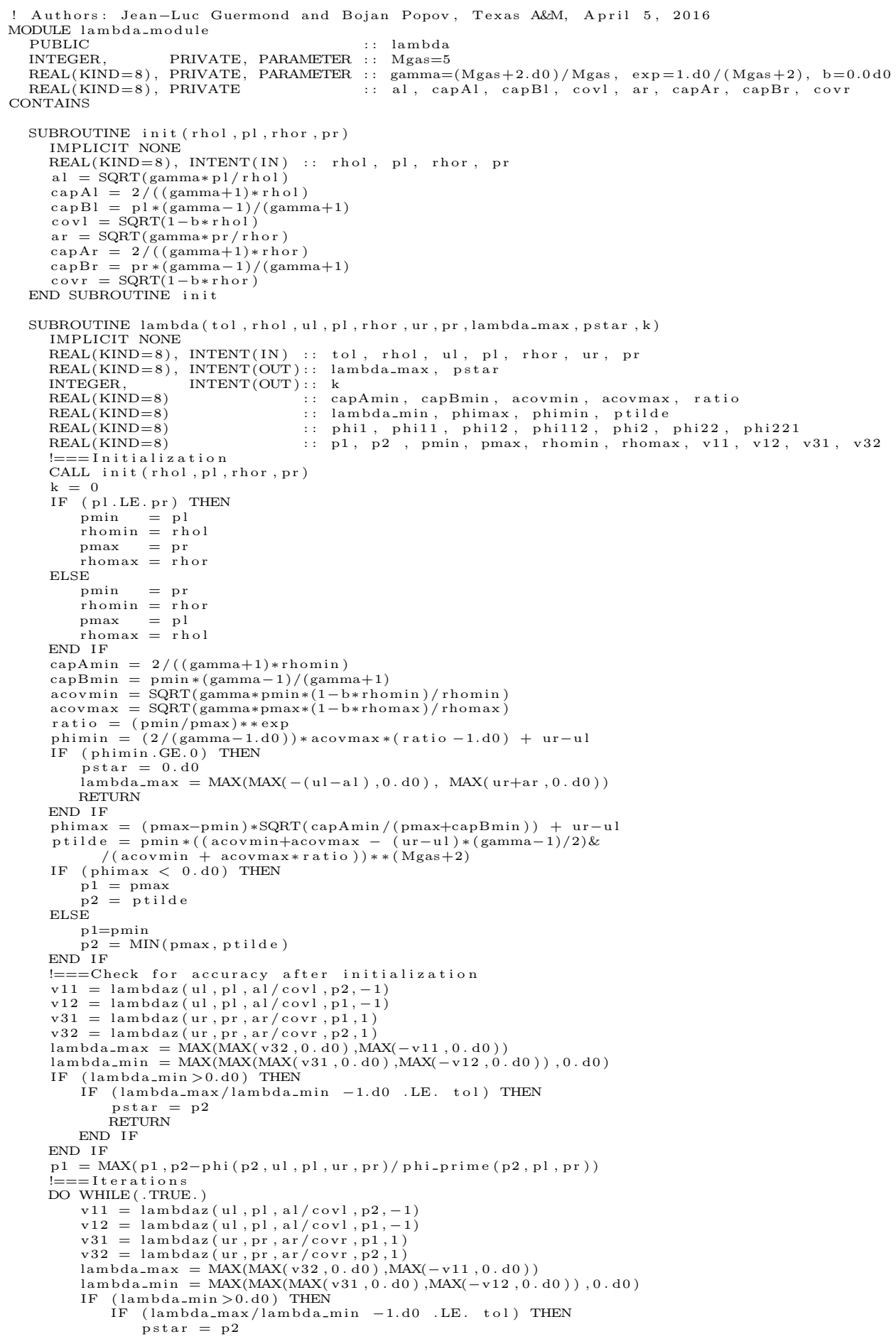




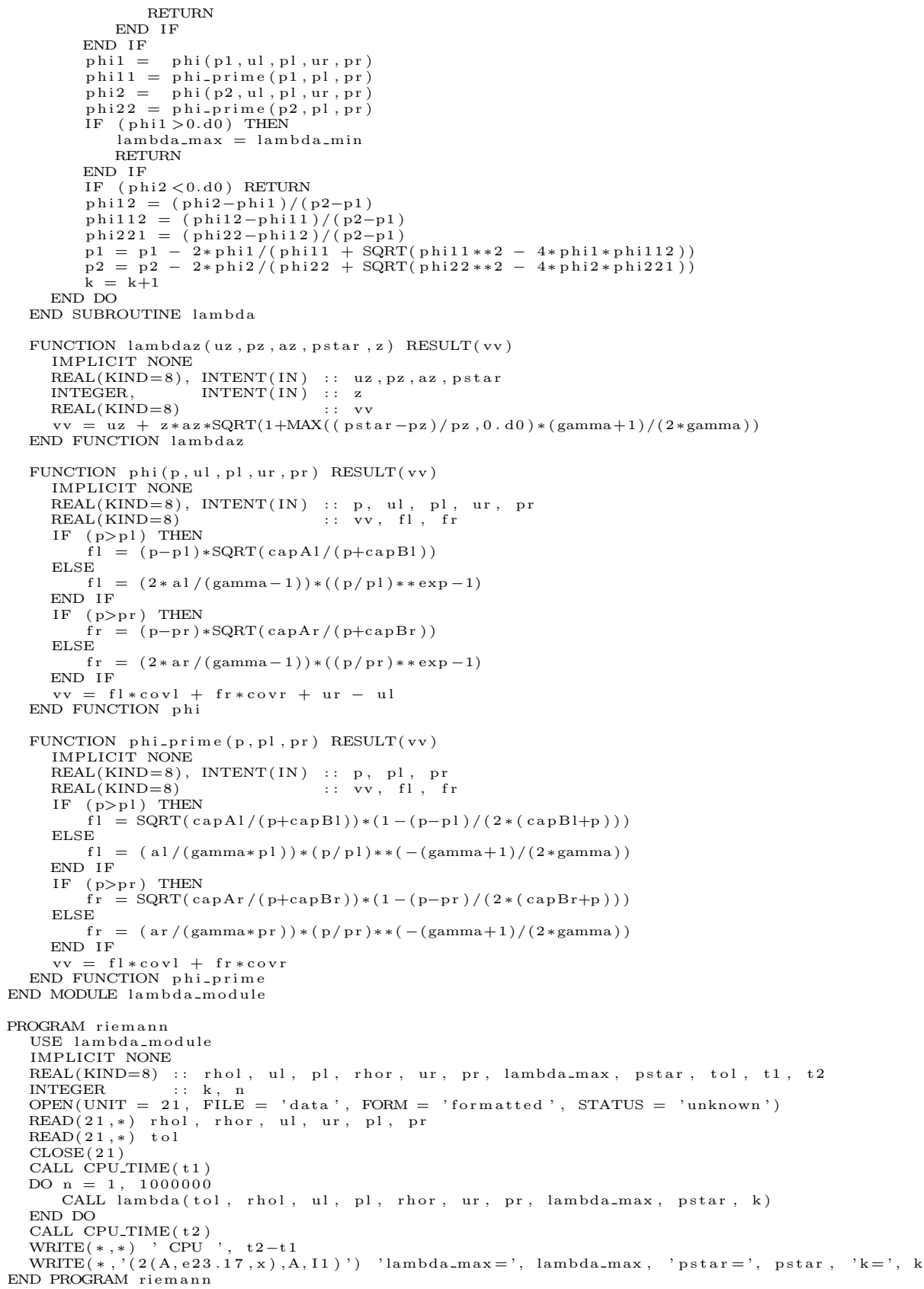

\section{References.}

[1] V. Baibuz, V. Zitserman, L. Golubushkin, and I. Malyshev. The covolume and equation of state of high-temperature real gases. Journal of Engineering Physics, 51(2):955-956, 1986.

[2] D. S. Balsara, M. Dumbser, and R. Abgrall. Multidimensional HLLC Riemann solver for unstructured meshes - with application to Euler and MHD flows. $J$. Comput. Phys., 261:172-208, 2014.

[3] F. Bouchut and T. Morales de Luna. Semi-discrete entropy satisfying approxi- 
mate Riemann solvers. The case of the Suliciu relaxation approximation. J. Sci. Comput., 41(3):483-509, 2009.

[4] A. Bressan. Hyperbolic systems of conservation laws, volume 20 of Oxford Lecture Series in Mathematics and its Applications. Oxford University Press, Oxford, 2000. The one-dimensional Cauchy problem.

[5] M. J. Castro, J. M. Gallardo, and A. Marquina. A class of incomplete Riemann solvers based on uniform rational approximations to the absolute value function. J. Sci. Comput., 60(2):363-389, 2014.

[6] A. J. Chorin. Random choice solution of hyperbolic systems. J. Computational Phys., 22(4):517-533, 1976.

[7] K. N. Chueh, C. C. Conley, and J. A. Smoller. Positively invariant regions for systems of nonlinear diffusion equations. Indiana Univ. Math. J., 26(2):373-392, 1977.

[8] P. Colella. Multidimensional upwind methods for hyperbolic conservation laws. J. Comput. Phys., 87(1):171-200, 1990.

[9] H. Frid. Maps of convex sets and invariant regions for finite-difference systems of conservation laws. Arch. Ration. Mech. Anal., 160(3):245-269, 2001.

[10] E. Godlewski and P.-A. Raviart. Numerical approximation of hyperbolic systems of conservation laws, volume 118 of Applied Mathematical Sciences. SpringerVerlag, New York, 1996.

[11] J.-L. Guermond and B. Popov. Invariant domains and first-order continuous finite element approximation for hyperbolic systems. 2015. arXiv:1509.07461, Submitted.

[12] J.-L. Guermond, B. Popov, L. Saavedra, and Y. Yang. Invariant domains preserving ale approximation of hyperbolic systems with continuous finite elements. SIAM J. Numer. Anal., 2016. in review.

[13] A. Harten, P. D. Lax, and B. van Leer. On upstream differencing and Godunovtype schemes for hyperbolic conservation laws. SIAM Rev., 25(1):35-61, 1983.

[14] D. Hoff. Invariant regions for systems of conservation laws. Trans. Amer. Math. Soc., 289(2):591-610, 1985.

[15] I. A. Johnston. The Noble-Abel equation of state: Thermodynamic derivations for ballistics modelling. Defence science and technology organization, Edinburgh (Australia) weapons system div., No. DSTO-TN-0670, 2005.

[16] A. Kurganov and E. Tadmor. New high-resolution central schemes for nonlinear conservation laws and convection-diffusion equations. J. Comput. Phys., 160(1): 241-282, 2000.

[17] A. Kurganov, S. Noelle, and G. Petrova. Semidiscrete central-upwind schemes for hyperbolic conservation laws and Hamilton-Jacobi equations. SIAM J. Sci. Comput., 23(3):707-740 (electronic), 2001.

[18] P. D. Lax. Hyperbolic systems of conservation laws. II. Comm. Pure Appl. Math., 10:537-566, 1957.

[19] V. Rusanov. The calculation of the interaction of non-stationary shock waves and obstacles. USSR Computational Mathematics and Mathematical Physics, 1 (2):304 - 320, 1962.

[20] E. F. Toro. Riemann solvers and numerical methods for fluid dynamics. SpringerVerlag, Berlin, third edition, 2009. A practical introduction.

[21] E. F. Toro and V. A. Titarev. Derivative Riemann solvers for systems of conservation laws and ADER methods. J. Comput. Phys., 212(1):150-165, 2006. 